\title{
EXPLICIT COERCIVITY ESTIMATES FOR THE LINEARIZED BOLTZMANN AND LANDAU OPERATORS
}

\author{
CLÉMENT MOUHOT
}

\begin{abstract}
We prove explicit coercivity estimates for the linearized Boltzmann and Landau operators, for a general class of interactions including any inversepower law interactions, and hard spheres. The functional spaces of these coercivity estimates depend on the collision kernel of these operators. They cover the spectral gap estimates for the linearized Boltzmann operator with Maxwell molecules, improve these estimates for hard potentials, and are the first explicit coercivity estimates for soft potentials (including in particular the case of Coulombian interactions). We also prove a regularity property for the linearized Boltzmann operator with non locally integrable collision kernels, and we deduce from it a new proof of the compactness of its resolvent for hard potentials without angular cutoff.
\end{abstract}

Mathematics Subject Classification (2000): 76P05 Rarefied gas flows, Boltzmann equation [See also 82B40, 82C40, 82D05].

Keywords: coercivity estimates, linearized Boltzmann operator, linearized Landau operator, quantitative, soft potentials, hard potentials, spectral gap.

\section{Contents}

\begin{tabular}{|lr|}
\hline 1. $\quad$ Introduction and main results & 1 \\
\hline 2. The linearized Boltzmann operator & 12 \\
\hline 3. The linearized Landau operator & 23 \\
\hline References & 30 \\
\hline
\end{tabular}

\section{INTRODUCTION AND MAIN RESULTS}

This paper is devoted to the study of the linearized Boltzmann and Landau collision operators. In this work we shall obtain new quantitative coercivity estimates for these operators. Before we explain our methods and results in more details, let us introduce the problem in a precise way. 
1.1. The models. The Boltzmann equation describes the behavior of a dilute gas when the only interactions taken into account are binary elastic collisions. It reads in $\mathbb{R}^{N}(N \geq 2)$

$$
\frac{\partial f}{\partial t}+v \cdot \nabla_{x} f=Q^{\mathcal{B}}(f, f)
$$

where $f(t, x, v)$ stands for the time-dependent probability density of particles in the phase space. The $N$-dimensional Boltzmann operator $Q^{\mathcal{B}}$ is a quadratic operator, which is local in $(t, x)$. The time and position are only parameters and therefore shall be omitted in the sequel: the functional estimates proved in this paper are all local in $(t, x)$. This operator acts on $f(v)$ by

$$
Q^{\mathcal{B}}(f, f)(v)=\int_{\mathbb{R}^{N} \times \mathbb{S}^{N-1}} B\left(\left|v-v_{*}\right|, \cos \theta\right)\left[f_{*}^{\prime} f^{\prime}-f_{*} f\right] d v_{*} d \sigma
$$

where we have used the shorthand $f=f(v), f_{*}=f\left(v_{*}\right), f^{\prime}=f\left(v^{\prime}\right), f_{*}^{\prime}=f\left(v_{*}^{\prime}\right)$. In this formula, $v^{\prime}, v_{*}^{\prime}$ and $v, v_{*}$ are the velocities of a pair of particles before and after collision, they are related by

$$
v^{\prime}=\left(v+v_{*}\right) / 2+\left(\left|v-v_{*}\right| / 2\right) \sigma, \quad v_{*}^{\prime}=\left(v+v_{*}\right) / 2-\left(\left|v-v_{*}\right| / 2\right) \sigma .
$$

The collision kernel $B$ is a non-negative function which only depends on the relative velocity $\left|v-v_{*}\right|$ and the deviation angle $\theta$ through $\cos \theta=k \cdot \sigma$ where $k=(v-$ $\left.v_{*}\right) /\left|v-v_{*}\right|$.

In the case of long-distance interactions, collisions occur mostly for very small $\theta$. When all collisions become concentrated on $\theta=0$, one obtains by the so-called grazing collision limit asymptotic (see for instance [6, 15, 16, 18, 29, 5]) the Landau operator

$$
Q^{\mathcal{L}}(f, f)(v)=\nabla_{v} \cdot\left(\int_{\mathbb{R}^{N}} \mathbf{A}\left(v-v_{*}\right)\left[f_{*}(\nabla f)-f(\nabla f)_{*}\right] d v_{*}\right),
$$

with $\mathbf{A}(z)=|z|^{2} \Phi(|z|) \mathbf{P}(z), \Phi$ is a non-negative function, and $\mathbf{P}(z)$ is the orthogonal projection onto $z^{\perp}$, i.e.,

$$
(\mathbf{P}(z))_{i, j}=\delta_{i, j}-\frac{z_{i} z_{j}}{|z|^{2}}
$$

This operator is used for instance in models of plasma in the case of a Coulomb potential where $\Phi(|z|)=|z|^{-3}$ in dimension 3 (for more details see [32, Chapter 1, Section 1.7] and the references therein). Indeed for Coulombian interactions the Boltzmann collision operator does not make sense anymore (see [31, Annex I, Appendix]). 
Boltzmann and Landau collision operators have the fundamental properties of preserving mass, momentum and energy $\left(Q\right.$ denotes $Q^{\mathcal{B}}$ or $\left.Q^{\mathcal{L}}\right)$

$$
\int_{\mathbb{R}^{N}} Q(f, f) \phi(v) d v=0, \quad \phi(v)=1, v,|v|^{2} .
$$

Moreover they satisfy well-known Boltzmann's $H$ theorem, which writes formally

$$
-\frac{d}{d t} \int_{\mathbb{R}^{N}} f \log f d v=-\int_{\mathbb{R}^{N}} Q(f, f) \log (f) d v \geq 0 .
$$

The functional $-\int f \log f$ is the entropy of the solution. The $H$ theorem implies formally that any equilibrium distribution, i.e., any distribution which maximizes the entropy, has the form of a locally Maxwellian distribution

$$
M(\rho, u, T)(v)=\frac{\rho}{(2 \pi T)^{N / 2}} \exp \left\{-\frac{|u-v|^{2}}{2 T}\right\}
$$

where $\rho, u, T$ are the mass, momentum and temperature of the gas

$$
\rho=\int_{\mathbb{R}^{N}} f(v) d v, \quad u=\frac{1}{\rho} \int_{\mathbb{R}^{N}} v f(v) d v, \quad T=\frac{1}{N \rho} \int_{\mathbb{R}^{N}}|u-v|^{2} f(v) d v .
$$

For further details on the physical background and derivation of the Boltzmann and Landau equations we refer to [12, 13, 24, 32].

1.2. Linearization. Consider the linearization process $f=M(1+h)$ around the Maxwellian equilibrium state denoted by $M$. It yields the linearized Boltzmann operator

$$
L^{\mathcal{B}} h(v)=\int_{\mathbb{R}^{N} \times \mathbb{S}^{N-1}} B\left(\left|v-v_{*}\right|, \cos \theta\right) M\left(v_{*}\right)\left[h_{*}^{\prime}+h^{\prime}-h_{*}-h\right] d v_{*} d \sigma,
$$

with (up to a normalization and without restriction) $M(v)=e^{-|v|^{2}}$. The normalization corresponds to the choice of mass $\pi^{N / 2}$, momentum 0 and temperature $1 / 2$. For the sake of simplicity we shall give all the statements and proofs under the normalization. It is explained briefly in Subsection 1.6 how to obtain the dependency of the estimates we shall establish in terms of the mass, momentum and energy of the equilibrium in the importance case where the collision kernel depends on the relative kernel in a polynomial way.

The operator $L^{\mathcal{B}}$ is self-adjoint on the Hilbert space $L^{2}(M)$, which is defined by the general convention ( $W$ is any positive weight function)

$$
L^{2}(W)=\left\{h: \mathbb{R}^{N} \rightarrow \mathbb{R} \text { measurable s. t. }\|h\|_{L^{2}(W)}^{2}:=\int_{\mathbb{R}^{N}} h(v)^{2} W(v) d v<+\infty\right\} .
$$


Since we shall need it in the sequel, we define similarly

$$
\begin{array}{r}
H^{1}(W)=\left\{h: \mathbb{R}^{N} \rightarrow \mathbb{R} \text { measurable s. t. }\|h\|_{H^{1}(W)}^{2}:=\int_{\mathbb{R}^{N}} h(v)^{2} W(v) d v\right. \\
\left.+\int_{\mathbb{R}^{N}}|\nabla h(v)|^{2} W(v) d v<+\infty\right\} .
\end{array}
$$

The Dirichlet form of $-L^{\mathcal{B}}$ in this space satisfies

$$
\begin{aligned}
D^{\mathcal{B}}(h) & =-\left(h, L^{\mathcal{B}} h\right)_{L^{2}(M)} \\
& =\frac{1}{4} \int_{\mathbb{R}^{N} \times \mathbb{R}^{N} \times \mathbb{S}^{N-1}} B\left(\left|v-v_{*}\right|, \cos \theta\right)\left[h_{*}^{\prime}+h^{\prime}-h_{*}-h\right]^{2} M M_{*} d v d v_{*} d \sigma .
\end{aligned}
$$

It is non-negative, which implies that the spectrum of $L^{\mathcal{B}}$ in $L^{2}(M)$ is included in $\mathbb{R}_{-}$. As it shall be useful in the sequel for the study of $L^{\mathcal{B}}$, we define the collision frequency (in $[0,+\infty])$ by

$$
\nu(v)=\int_{\mathbb{R}^{N} \times \mathbb{S}^{N-1}} B\left(\left|v-v_{*}\right|, \cos \theta\right) M\left(v_{*}\right) d v_{*} .
$$

The same linearization process yields the linearized Landau operator

$$
L^{\mathcal{L}} h(v)=M(v)^{-1} \nabla_{v} \cdot\left(\int_{v_{*} \in \mathbb{R}^{N}} \mathbf{A}\left(v-v_{*}\right)\left[(\nabla h)-(\nabla h)_{*}\right] M M_{*} d v_{*}\right),
$$

which is self-adjoint on $L^{2}(M)$, and whose Dirichlet form satisfies

$$
\begin{aligned}
D^{\mathcal{L}}(h) & =-\left(h, L^{\mathcal{L}} h\right)_{L^{2}(M)} \\
& =\frac{1}{2} \int_{\mathbb{R}^{N}} \int_{\mathbb{R}^{N}} \Phi\left(v-v_{*}\right)\left|v-v_{*}\right|^{2}\left\|\mathbf{P}\left(v-v_{*}\right)\left[(\nabla h)-(\nabla h)_{*}\right]\right\|^{2} M M_{*} d v_{*} d v .
\end{aligned}
$$

It is also non-negative, which implies that the spectrum of $L^{\mathcal{L}}$ in $L^{2}(M)$ is included in $\mathbb{R}_{-}$. The null space of the two operators $L^{\mathcal{L}}$ and $L^{\mathcal{B}}$ is

$$
N\left(L^{\mathcal{B}}\right)=N\left(L^{\mathcal{L}}\right)=\operatorname{Span}\left\{1, v_{1}, \ldots, v_{N},|v|^{2}\right\}
$$

(note that it is independent on the collision kernel). These two properties - the fact that the time-derivative of the $L^{2}(M)$ norm is negative and the fact that the only functions which cancel this derivative are the collision invariants - correspond to the $H$ theorem at the linearized level. We denote by $\Pi$ the orthogonal projection on this null space in $L^{2}(M)$. 


\subsection{Assumptions on the collision kernel.}

- In the case of the linearized Boltzmann operator, $B$ takes the product form

$$
B\left(\left|v-v_{*}\right|, \cos \theta\right)=\Phi\left(\left|v-v_{*}\right|\right) b(\cos \theta),
$$

where $\Phi$ and $b$ are non-negative functions.

- The kinetic part $\Phi$ is bounded by a power-law:

$$
\forall r \geq 0, \quad C_{\Phi} r^{\gamma} \leq \Phi(r) \leq C_{\Phi}^{\prime} r^{\gamma} .
$$

where $\gamma \in(-N, 1]$ for the linearized Boltzmann operator, or $\gamma \in[-N, 1]$ for the linearized Landau operator, and $C_{\Phi}, C_{\Phi}^{\prime}>0$ are some constants.

- In the case of the linearized Boltzmann operator, the angular part $b$ satisfies

$$
C_{b}=\inf _{\sigma_{1}, \sigma_{2} \in \mathbb{S}^{N-1}} \int_{\sigma_{3} \in \mathbb{S}^{N-1}} \min \left\{b\left(\sigma_{1} \cdot \sigma_{3}\right), b\left(\sigma_{2} \cdot \sigma_{3}\right)\right\} d \sigma_{3}>0 .
$$

This quite unusual (but satisfied for all physical models) assumption was defined and used in the work [7] in order to obtain explicit spectral gap estimates for hard potentials.

- In the particular case of the linearized Boltzmann operator with a non locally integrable collision kernel, we shall assume the following control in order to derive coercivity estimates in Sobolev spaces

$$
\forall \theta \in(0, \pi], \quad \frac{c_{b}}{\theta^{N-1+\alpha}} \leq b(\cos \theta) \leq \frac{c_{b}^{\prime}}{\theta^{N-1+\alpha}}
$$

for some constants $c_{b}, c_{b}^{\prime}>0$ and $\alpha \in[0,2)$ (note that assumption (1.4) implies straightforwardly assumption (1.3) $)$. The goal of this control is to measure the strength of the angular singularity, which is related to the regularity properties of the collision operator (see [4] for instance).

For the linearized Boltzmann operator, our assumptions cover in dimension 3 the hard spheres collision kernel $B\left(\left|v-v_{*}\right|, \cos \theta\right)=\operatorname{cst}\left|v-v_{*}\right|$ (which satisfies (1.1] 1.2. [1.3) ), and collision kernels deriving from interaction potentials behaving like inversepower laws (which satisfy (1.1,1.2,1.4) ). More precisely for an interaction potential $V(r)=\operatorname{cst} r^{-(s-1)}, B$ satisfies the assumptions (1.1]1.2 1.4) with the formulas $\gamma=$ $(s-5) /(s-1)$ and $\alpha=2 /(s-1)$ (see [12). One traditionally denotes by hard potentials the case $s>5$ (for which $0<\gamma<1$ ), Maxwell molecules the case $s=5$ (for which $\gamma=0$ ), and soft potentials the case $2<s<5$ (for which $-N<\gamma<0$ ). In the case of an angular cutoff, $b$ is assumed artificially to be integrable.

For the linearized Landau operator, our assumptions are satisfied for any collision kernel $\Phi\left(\left|v-v_{*}\right|\right)=\left|v-v_{*}\right|^{\gamma},-N \leq \gamma \leq 1$, including in particular in dimension 3 the most important case of the linearized Landau-Coulomb operator when $\gamma=-3$ (corresponding to Coulombian interactions between charged particles). By analogy 
with the linearized Boltzmann operator, one traditionally denotes by hard potentials the case $\gamma>0$, Maxwell molecules the case $\gamma=0$, and soft potentials the case $-N \leq \gamma<0$.

Remark: The assumption (1.1) is made for a sake of simplicity. Indeed, one could easily adapt the proofs in Section 2 to relax this assumption to upper and lower bounds on $B$ of the multiplicative type (1.1). For more general $B$ without decoupling between the arguments, more technical conditions would be needed to apply our strategy.

Let us comment on the domains $\mathcal{D}\left(L^{\mathcal{B}}\right)$ and $\mathcal{D}\left(L^{\mathcal{L}}\right)$ of the linearized Boltzmann and Landau operators according to the previous assumptions:

- In the case of the linearized Boltzmann collision operators with locally integrable collision kernel, it is straightforward that the domain is $L^{2}\left(\langle v\rangle \gamma^{+} M\right)$ where $\gamma^{+}$is the nonnegative part of $\gamma$. Indeed as discussed in Section 2 it is well-known (see [19] for instance) that $L^{\mathcal{B}}$ then splits between a bounded part and a multiplicative part, which is the multiplication operator by the collision frequency.

- In the case of the linearized Landau operator, obvious estimates show that the domain contains $H^{1}\left(\langle v\rangle^{\gamma+2} M\right) \cap L^{2}(M)$. The maximal domain is slightly bigger than this space, it is obtained in [20]:

$h \in L^{2}(M)$ s.t. $\|h\|_{L^{2}\left(\langle v\rangle^{\gamma+2} M\right)}+\left\|\mathbf{P}(v) \nabla_{v} h\right\|_{L^{2}\left(\langle v\rangle^{\gamma+2} M\right)}+\left\|(\operatorname{Id}-\mathbf{P}(v)) \nabla_{v} h\right\|_{L^{2}\left(\langle v\rangle^{\gamma} M\right)}<+\infty$

where $\mathbf{P}(v)$ is the orthogonal projection on $v^{\perp}$ defined above.

- In the case of the linearized Boltzmann operator with non locally integrable collision kernel, the question of characterizing the domain in terms of some known functional spaces is still open. However one can prove easily (using a Taylor expansion and the kind of changes of variables of Subsection [2.3) that

$$
\left\|L^{\mathcal{B}} h\right\|_{L^{2}(M)} \leq C\|h\|_{H^{1}\left(\langle v\rangle(\gamma+2){ }^{+} M\right)}
$$

which shows that the domain includes $H^{1}\left(\langle v\rangle^{(\gamma+2)^{+}} M\right)$.

1.4. Motivation. We refer to [7] and the references therein for a discussion about the interest of spectral gap estimates for the linearized Boltzmann and Landau operators and some review. Let us just recall that since Grad [19], spectral gap estimates is known to exist at least for Maxwell molecules, hard potentials (with or without angular cutoff) and hard spheres. However, apart for the case of Maxwell molecules, for which the linearized Boltzmann operator is diagonalized explicitly in 34, 9], the classical proof of the existence of a spectral gap by Grad is based on non-constructive arguments and does not provide any estimate. In [7, it is given a new method to obtain explicit spectral gap estimates for any $\gamma \geq 0$ (including the so-called hard potentials - with or without angular cutoff - and hard spheres cases). 
This method relies on a geometrical argument on the whole collision operator, with no need of splitting or angular cutoff assumptions. The result is also extended in the same work to the linearized Landau operator (for $\gamma \geq 0$ ) by a grazing collision asymptotic.

As for soft potentials in dimension $N=3$ with $-1<\gamma<0$ and angular cutoff, it was proved in 10, that the Boltzmann linearized operator has no spectral gap. But if one allows a loss on the algebraic weight of the norm, it was proved in [17] (in dimension $N=3$ with $-2<\gamma<0$ and angular cutoff) a "degenerated spectral gap" result of the form

$$
D^{\mathcal{B}}(h) \geq C\left\|[h-\Pi(h)]\langle v\rangle^{\gamma / 2}\right\|_{L^{2}(M)}^{2}
$$

where we have denoted $\langle\cdot\rangle=\sqrt{1+|\cdot|^{2}}$. The proof was based on Weyl's Theorem about compact perturbation of the essential spectrum, and it leads to non explicit constants. The inequality (1.5) was then extended to the full range $-N<-\gamma<0$ in [21] by a similar non-constructive approach.

In this work we shall extend and complete the works [10, 17, 21] and [7] by

- giving a constructive proof of (1.5) for soft potentials, with explicit estimate on the constant (note that all through this paper the word "explicit" refers to the fact that all steps in our proofs are constructive and explicit computations of the constants could be extracted from them);

- extending it to hard potentials $(\gamma>0)$ (note that for hard potentials this estimate is stronger than the usual spectral gap estimate);

- extending this approach to the linearized Landau operator by proving coercivity estimates in $H^{1}$ with a weight corresponding to the collision kernel;

- giving a coercivity result in local Sobolev spaces for the linearized Boltzmann operator with a non locally integrable collision kernel, and discussing the consequence on its spectrum.

There are the first explicit coercivity estimates for weak collision interaction models such as the linearized Boltzmann for soft potentials or the linearized LandauCoulomb operator (that is the linearized Landau operator in the case of Coulombian interactions). These estimates are crucial for instance for the construction of a quantitative perturbative theory near equilibrium and for obtaining quantitative rates of convergence to equilibrium, which shall be discussed in a forthcoming work.

In addition, the regularity coercivity estimates derived for the linearized Boltzmann operator allow to give a new simpler proof of the compactness of the resolvent for hard potentials without angular cutoff (which implies that the spectrum is purely discrete in this case). This revisits partly results in [28, 23. We recall that in [28], it was proved that the linearized Boltzmann operator has compact resolvent for interaction potentials $V(r)=\operatorname{cst} r^{-(s-1)}$ with $3<s<+\infty$ in dimension $N=3$. 
As a byproduct, our results give a new proof of this fact in the case $s \geq 5$ (hard potentials).

1.5. Statements of the results. We now state our main results:

Theorem 1.1 (The linearized Boltzmann operator). Under the assumptions (1.1), (1.2), (1.3), the linearized Boltzmann operator $L^{\mathcal{B}}$ with collision kernel $B=\Phi b$ satisfies

$$
\forall h \in \mathcal{D}\left(L^{\mathcal{B}}\right), \quad D^{\mathcal{B}}(h) \geq C_{\gamma}^{\mathcal{B}}\left\|[h-\Pi(h)]\langle v\rangle^{\gamma / 2}\right\|_{L^{2}(M)}^{2},
$$

where $C_{\gamma}^{\mathcal{B}}$ is an explicit constant depending only on $\gamma, C_{\Phi}, C_{b}$, and the dimension $N$.

\section{Remarks:}

1. When the collision kernel is locally integrable, the collision frequency $\nu$ is finite, bounded from below, and asymptotically equivalent to $\langle v\rangle^{\gamma}$, and the estimate (1.6) can be written in the following form

$$
D^{\mathcal{B}}(h) \geq \bar{C}_{\gamma}^{\mathcal{B}}\left\|[h-\Pi(h)] \nu^{1 / 2}\right\|_{L^{2}(M)}^{2}
$$

for some explicit constant $\bar{C}_{\gamma}^{\mathcal{B}}>0$.

2. When the collision kernel is not locally integrable and $b$ satisfies (1.4), a natural conjecture would be that the estimate (1.6) improves into

$$
D^{\mathcal{B}}(h) \geq C_{\gamma, \alpha}^{\mathcal{B}}\left\|[h-\Pi(h)]\langle v\rangle^{\gamma / 2}\right\|_{H^{\alpha / 2}(M)}^{2},
$$

where $\alpha \in[0,2)$ is the order of angular singularity, defined in (1.4), and $H^{\alpha / 2}(M)$ is the Sobolev space defined by $H^{\alpha / 2}(M)=\left\{h \in L^{2}(M)\right.$ s. t. $\left.(1-\Delta)^{-\alpha / 4} h \in L^{2}(M)\right\}$. We were not able to obtain this coercivity estimate, however we give in the following theorem its consequence in terms of local regularity. We denote by $H_{\mathrm{loc}}^{\alpha / 2}$ the space of functions whose restriction to any compact set $K$ of $\mathbb{R}^{N}$ belongs to $H^{\alpha / 2}(K)=$ $\left\{h \in L^{2}(K)\right.$ s. t. $\left.(1-\Delta)^{-\alpha / 4} h \in L^{2}(K)\right\}$ (here $L^{2}(K)$ denotes the space of functions square integrable on $K$ ).

Theorem 1.2 (The linearized Boltzmann operator for long-range interactions). Under the assumptions (1.1), (1.2), (1.4), the linearized Boltzmann operator $L^{\mathcal{B}}$ with collision kernel $B=\Phi b$ satisfies (1.6) and

$$
\forall h \in \mathcal{D}\left(L^{\mathcal{B}}\right), \quad D^{\mathcal{B}}(h) \geq C_{\gamma, \alpha}^{\mathcal{B}}\|h-\Pi(h)\|_{H_{\mathrm{loc}}^{\alpha / 2}}^{2},
$$

where $C_{\gamma, \alpha}^{\mathcal{B}}$ is an explicit constant depending only on $\gamma, \alpha, C_{\Phi}, C_{b}, c_{b}$ and the dimension $N$. 


\section{Remarks:}

1. When $\gamma>0$ and $\alpha>0$, one can deduce straightforwardly from Theorem 1.2 that the operator $L^{\mathcal{B}}$ has compact resolvent, which implies that its spectrum is purely discrete in this case. Indeed let us pick any $\xi \in \mathbb{C}$ such that $L^{\mathcal{B}}-\xi$ is invertible (such $\xi$ exists since the operator is self-adjoint for instance), and let us denote by $R(\xi)=\left(L^{\mathcal{B}}-\xi\right)^{-1}$ the resolvent at this point. For any sequence $\left(g_{n}\right)_{n \geq 0}$ bounded in $L^{2}(M)$, we can define the sequence $h_{n}=R(\xi)\left(g_{n}\right)$ which is also bounded in $L^{2}(M)$ since the operator $R(\xi)$ is bounded. We have:

$$
\forall n \geq 0, \quad L^{\mathcal{B}}\left(h_{n}\right)=g_{n}+\xi h_{n}
$$

and so the sequence $L^{\mathcal{B}}\left(h_{n}\right)$ is bounded in $L^{2}(M)$. It follows that the sequence $D^{\mathcal{B}}\left(h_{n}\right)$ is bounded in $\mathbb{R}$, and we deduce from the coercivity estimates above that the sequence $\left(h_{n}\right)_{n \geq 0}$ is bounded in $L^{2}\left(\langle v\rangle^{\gamma} M\right) \cap H_{\mathrm{loc}}^{\alpha / 2}$. When $\alpha>0$ and $\gamma>0$, it implies that it has a cluster point in $L^{2}(M)$ by Rellich-Kondrachov compactness Theorem. Thus the operator $R(\xi)$ is compact. By classical arguments (see 22 for instance), it implies that the resolvent $R(\xi)$ is compact at every $\xi \in \mathbb{C}$ for which it is defined, and that the spectrum of $L^{\mathcal{B}}$ is purely discrete.

2. The case $\alpha=0$ could probably be treated in the same spirit as in Theorem 1.2 using for the coercivity estimate a functional space controlling logarithmic derivatives defined by the norm $\|h \log (1-\Delta) h\|_{L_{\text {loc }}^{2}}$. We expect the Remark 1 to extend to this case as well. The restriction $\gamma>0$ in Remark 1 seems more serious at first sight, since in the case $\gamma \leq 0$, the coercivity estimate from Theorem 1.1 does not forbid the loss of mass at infinity. However on one hand for $\gamma=0$ and $b$ non locally integrable, the explicit diagonalization of the linearized Boltzmann operator (see 9] for instance) shows that it has discrete spectrum (and compact resolvent) as well. On the other hand we shall give new improved coercivity estimates in weighted $L^{2}(M)$ spaces in the non-cutoff case in the forthcoming work [27, showing that this restriction can still be further relaxed.

3. Our proof covers the physical case of inverse power-law interaction potentials $V(r)=\operatorname{cst} r^{-(s-1)}$ with $s>5$ in dimension $N=3$ (for which $\gamma>0$ and $\alpha>0$ ). The limit case $s=5$ corresponds to Maxwell molecules (for which $\gamma=0$ and $\alpha>0$ ). It can be treated thanks to the explicit diagonalization for Maxwell molecules (see the previous Remark 2).

Concerning the linearized Landau operator we prove the 
Theorem 1.3 (The linearized Landau operator). Under assumptions (1.2), the linearized Landau operator $L^{\mathcal{L}}$ with collision kernel $\Phi$ satisfies

$\forall h \in \mathcal{D}\left(L^{\mathcal{L}}\right), \quad D^{\mathcal{L}}(h) \geq C_{\gamma}^{\mathcal{L}}\left(\|[h-\Pi(h)]\|_{H^{1}\left(\langle v\rangle^{\gamma} M\right)}^{2}+\left\|[h-\Pi(h)]\langle v\rangle^{1+\gamma / 2}\right\|_{L^{2}(M)}^{2}\right)$, where $C_{\gamma}^{\mathcal{L}}$ is an explicit constant depending only on $\gamma, C_{\Phi}$, and the dimension $N$.

\section{Remarks:}

1. It was already noticed (by non-constructive arguments) in 20] that the Dirichlet form of the linearized Landau operator controls the $L^{2}\left(\langle v\rangle^{\gamma+2} M\right)$ norm. Moreover in [20] it is given (still by non-constructive arguments) estimates of the form

$$
D^{\mathcal{L}}(h) \geq C\left(\|h\|_{L^{2}\left(\langle v\rangle^{\gamma+2} M\right)}^{2}+\left\|\mathbf{P}(v) \nabla_{v} h\right\|_{L^{2}\left(\langle v\rangle^{\gamma+2} M\right)}^{2}+\left\|(\mathrm{Id}-\mathbf{P}(v)) \nabla_{v} h\right\|_{L^{2}\left(\langle v\rangle^{\gamma} M\right)}^{2}\right)
$$

which use on the right-hand side some norm slightly stronger than the one in (1.8). The proof of explicit coercivity estimates for this stronger norm shall be given in the forthcoming work [27].

2. By a similar argument as for the linearized Boltzmann operator with a non locally integrable collision kernel, when $\gamma>-2$, we straightforwardly deduce from Theorem [1.3 that the linearized Landau operator has compact resolvent and thus a purely discrete spectrum. Indeed if one considers a sequence $\left(h_{k}\right)_{k \geq 0}$ which is bounded in $L^{2}(M)$ and such that $\left(L^{\mathcal{L}}\left(h_{k}\right)\right)_{k \geq 0}$ is bounded in $L^{2}(M)$, the coercivity estimate (1.8) implies that this sequence is bounded in $H_{\mathrm{loc}}^{1} \cap L^{2}\left(\langle v\rangle^{2+\gamma} M\right)$. Since $2+\gamma>0$ when $\gamma>-2$, this implies by Rellich-Kondrachov Theorem that the sequence has a cluster point in $L^{2}(M)$.

\subsection{Dependence of the constants in the coercivity estimates according to the equilibrium.}

Straightforward computations show that the Dirichlet form with mass $\rho$, momentum $u$ and temperature $T$ satisfies (with obvious notation)

$$
D_{\rho, u, T}^{\mathcal{B}}(h)=\frac{\rho^{2}}{\pi^{N}}(2 T)^{\gamma / 2} D^{\mathcal{B}}(h(u+\sqrt{2 T} v))
$$

in the Boltzmann case, and

$$
D_{\rho, u, T}^{\mathcal{L}}(h)=\frac{\rho^{2}}{\pi^{N}}(2 T)^{\gamma / 2+1} D^{\mathcal{L}}(h(u+\sqrt{2 T} v))
$$

in the Landau case. Moreover we have for the norms involved in our estimates:

$$
\|h\|_{L^{2}\left(\langle v\rangle \gamma M_{\rho, u, T}\right)}=\frac{\rho}{\pi^{N / 2}}(2 T)^{\gamma / 2}\|h(u+\sqrt{2 T} v)\|_{L^{2}(\langle v\rangle \gamma M)}
$$


for the norms involved in the Boltzmann case (for the $H_{\mathrm{loc}}^{\alpha / 2}$ space the dependency cannot be written with such a simple formula) and

$$
\|h\|_{H^{1}\left(\langle v\rangle^{\gamma} M_{\rho, u, T}\right)}=\frac{\rho}{\pi^{N / 2}}(2 T)^{\gamma / 2}\|h(u+\sqrt{2 T} v)\|_{L^{2}\left(\langle v\rangle^{\gamma} M\right)}
$$

and

$$
\|h\|_{L^{2}\left(\langle v\rangle^{\gamma+2} M_{\rho, u, T}\right)}=\frac{\rho}{\pi^{N / 2}}(2 T)^{\gamma / 2+1}\|h(u+\sqrt{2 T} v)\|_{L^{2}\left(\langle v\rangle^{\gamma} M\right)}
$$

for the norms involved in the Landau case. This explains how to modify the constants in Theorems 1.1] and 1.3 for a general equilibrium.

For instance for an equilibrium with mass $\rho$, momentum $u$ and temperature $T$, the constant in Theorem 1.1 has to be multiplied by some factor $\rho / \pi^{N / 2}$. This constant is independent of the momentum, which is a well-known consequence of the translation invariance of the Boltzmann equation. It is also independent of the temperature, which is due to the fact that our polynomial weight matches exactly the polynomial dependency of the collision kernel in terms of the relative velocity.

1.7. Method of proof. In the case of hard potentials, the idea is to decompose the operator between a part satisfying the desired coercivity estimate and a bounded part, and use the spectral gap estimates. This argument is reminiscent of an argument of Grad [19, Section 5] used to study the behavior at $v \rightarrow \infty$ of the eigenvectors of the linearized Boltzmann operator for hard potentials, and it was already noticed in [8]. Nevertheless it is the first time that it is used to obtain explicit estimates (thanks to the results in [7]). The same idea, combined with a suitable Poincaré inequality, is applied to the linearized Landau operator.

For soft potentials we decompose the Dirichlet form according to the modulus of the relative velocity. Combined with technical estimates on the non-local part of the linearized collision operators and the coercivity estimates from the Maxwell case, it enables to reconstruct a lower bound with the appropriate weight. The proof for the linearized Landau is strongly guided by the previous study of the Boltzmann case, which helps to identify relevant estimates.

Finally the proof of the coercivity estimates in local Sobolev spaces for the linearized Boltzmann operator with a non locally integrable collision kernel is inspired by the previous works [26, 30, 4] on the full non-linear collision operator, and by our study of the linearized Landau operator. Indeed the suitable decomposition of $L^{\mathcal{B}}$ for non locally integrable collision kernels (for which the usual Grad's splitting does not make sense anymore) is directly readable on the linearized Landau operator: the part which becomes the diffusion part in the grazing collision limit is the part which enjoys a coercivity property in local Sobolev spaces, and the part which becomes the bounded part in the grazing collision limit is the part which is bounded thanks to the "cancellation lemmas" (which we borrow from [30, 4]). Let us also mention 
that, as indicated by one of the anonymous referee, a decomposition in the same spirit was proposed in the papers [2, 3].

1.8. Plan of the paper. Section 2 is devoted to the linearized Boltzmann operator: it contains the proof of Theorem 1.1. divided into two parts, for hard and then soft potentials, and then the proof of Theorem 1.2. Section 3 is devoted to the linearized Landau operator: it contains the proof of Theorem 1.3, divided into hard and soft potentials again.

\section{THE LINEARIZED BOLTZMANN OPERATOR}

In this section and the next one, the constants which are only internal to a proof shall be denoted $C_{1}, C_{2}, \ldots$ if they are referred to inside the proof, or simply $C$ if not.

2.1. Hard potentials. Notice that the case $\gamma=0$ of Theorem 1.1 is already proved by the explicit estimates of the Maxwell case, see [9]. Hence we assume that $\gamma>$ 0 and we pick $h \in L^{2}(M)$ orthogonal to the null space of $L^{\mathcal{B}}$. First using the minoration of $b$ (1.3) we reduce to the (cutoff case) where $b \equiv 1$ by [7, Lemma 2.1], and using the assumption (1.2) we reduce to the case $\Phi(z)=z^{\gamma}$.

We consider the decomposition

$$
L^{\mathcal{B}}=K^{\mathcal{B}}-A^{\mathcal{B}}
$$

with

$$
K^{\mathcal{B}} h(v)=\int_{\mathbb{R}^{N} \times \mathbb{S}^{N-1}} B\left(\left|v-v_{*}\right|, \cos \theta\right) M\left(v_{*}\right)\left[h_{*}^{\prime}+h^{\prime}-h_{*}\right] d v_{*} d \sigma
$$

and

$$
A^{\mathcal{B}} h(v)=\left(\int_{\mathbb{R}^{N} \times \mathbb{S}^{N-1}} B\left(\left|v-v_{*}\right|, \cos \theta\right) M\left(v_{*}\right) d v_{*} d \sigma\right) h(v) .
$$

Then we use Grad computations [19, Sections 2, 3, 4] to obtain that $K^{\mathcal{B}}$ is a (compact) bounded operator (with explicit bound $C_{K}^{\mathcal{B}}$ ) and $A^{\mathcal{B}}$ is the multiplication operator by the collision frequency $\nu$, given here by

$$
\nu(v)=\left|\mathbb{S}^{N-1}\right| \int_{\mathbb{R}^{N}}\left|v-v_{*}\right|^{\gamma} M\left(v_{*}\right) d v_{*} .
$$

On one hand we have straightforwardly

$$
\int_{\mathbb{R}^{N}}\left(A^{\mathcal{B}} h\right) h M d v \geq C_{1}\left\|h\langle v\rangle^{\gamma / 2}\right\|_{L^{2}(M)}^{2}
$$


with $C_{1}>0$ depending on $\gamma$. On the other hand we know by [7, Theorem 1.1] that there is an explicit constant $C_{2}>0$ such that

$$
D^{\mathcal{B}}(h)=-\int_{\mathbb{R}^{N}}\left(L^{\mathcal{B}} h\right) h M d v \geq C_{2}\|h\|_{L^{2}(M)}^{2} .
$$

We deduce then that

$$
\begin{aligned}
&\left\|h\langle v\rangle^{\gamma / 2}\right\|_{L^{2}(M)}^{2} \leq C_{1}^{-1} \int_{\mathbb{R}^{N}}\left(A^{\mathcal{B}} h\right) h M d v \\
& \leq C_{1}^{-1}\left[-\int_{\mathbb{R}^{N}}\left(L^{\mathcal{B}} h\right) h M d v+\int_{\mathbb{R}^{N}}\left(K^{\mathcal{B}} h\right) h M d v\right] \\
& \leq C_{1}^{-1}\left[D^{\mathcal{B}}(h)+C_{K}^{\mathcal{B}}\|h\|_{L^{2}(M)}^{2}\right] \\
& \quad \leq C_{1}^{-1}\left[1+C_{K}^{\mathcal{B}} C_{2}^{-1}\right] D^{\mathcal{B}}(h)
\end{aligned}
$$

which concludes the proof of Theorem 1.1] in the case $\gamma>0$.

2.2. Soft potentials. We suppose now that $\gamma<0$ and we pick $h \in L^{2}(M)$ orthogonal to the null space of $L^{\mathcal{B}}$. First using (1.3) we reduce to the (cutoff case) where $b \equiv 1$ by using [7. Lemma 2.1] again (this lemma is independent on the particular form of $\Phi)$, and using (1.2) we reduce to the case $\Phi(z)=\min \left\{z^{\gamma}, 1\right\}$.

Step 1. We need first a technical lemma on $K^{\mathcal{B}}$, in the case of Maxwell molecules. We define

$$
K_{R}^{\mathcal{B}} h(v)=\int_{\mathbb{R}^{N} \times \mathbb{S}^{N-1}} \mathbf{1}_{\left\{\left|v-v_{*}\right| \geq R\right\}} M\left(v_{*}\right)\left[h_{*}^{\prime}+h^{\prime}-h_{*}\right] d v_{*} d \sigma .
$$

Then

Lemma 2.1. The bounded operator $K_{R}^{\mathcal{B}}$ satisfies

$$
\left\|K_{R}^{\mathcal{B}}\right\|_{L^{2}(M)} \stackrel{R \rightarrow \infty}{\longrightarrow} 0
$$

with explicit rate (here \|\|$\cdot\|\|_{L^{2}(M)}$ denotes the usual operator norm on $L^{2}(M)$ ).

Proof of Lemma 2.1. First we decompose $K_{R}^{\mathcal{B}}=T_{R}-U_{R}$ with (using the change of variable $\sigma \rightarrow-\sigma$ that exchanges $v^{\prime}$ and $v_{*}^{\prime}$ )

$$
T_{R} h(v)=2 \int_{\mathbb{R}^{N} \times \mathbb{S}^{N-1}} \mathbf{1}_{\left\{\left|v-v_{*}\right| \geq R\right\}} M\left(v_{*}\right) h^{\prime} d \sigma d v_{*}
$$

and

$$
U_{R} h(v)=\int_{\mathbb{R}^{N} \times \mathbb{S}^{N-1}} \mathbf{1}_{\left\{\left|v-v_{*}\right| \geq R\right\}} M\left(v_{*}\right) h_{*} d \sigma d v_{*}
$$


The proof for $U_{R}$ is straightforward:

$$
\left\|U_{R} h\right\|_{L^{2}(M)} \leq\left|\mathbb{S}^{N-1}\right|^{1 / 2}\|h\|_{L^{2}(M)}\left(\int_{\mathbb{R}^{N} \times \mathbb{R}^{N}} \mathbf{1}_{\left\{\left|v-v_{*}\right| \geq R\right\}} M M_{*} d v d v_{*}\right)^{1 / 2}
$$

which gives the convergence to 0 for the operator norm with the rate.

The term $T_{R}$ is more tricky to handle. First, using $M M_{*}=M^{\prime} M_{*}^{\prime}$, we write it as

$$
T_{R} h(v)=2 M(v)^{-1 / 2} \int_{\mathbb{R}^{N} \times \mathbb{S}^{N-1}} \mathbf{1}_{\left\{\left|v-v_{*}\right| \geq R\right\}}\left(M^{\prime}\right)^{1 / 2} h^{\prime}\left(M_{*}\right)^{1 / 2}\left(M_{*}^{\prime}\right)^{1 / 2} d \sigma d v_{*} .
$$

Then we use the bound

$$
\mathbf{1}_{\left\{\left|v-v_{*}\right| \geq R\right\}} \leq \mathbf{1}_{\left\{\left|v-v^{\prime}\right| \geq R / \sqrt{2}\right\}}+\mathbf{1}_{\left\{\left|v-v_{*}^{\prime}\right| \geq R / \sqrt{2}\right\}}
$$

which yields a corresponding decomposition $\left|T_{R} h\right| \leq T_{R}^{1} h+T_{R}^{2} h$ with

$$
\left\{\begin{array}{l}
T_{R}^{1} h(v)=2 M^{-1 / 2} \int_{\mathbb{R}^{N} \times \mathbb{S}^{N-1}} \mathbf{1}_{\left\{\left|v-v^{\prime}\right| \geq R / \sqrt{2}\right\}}\left(M^{\prime}\right)^{1 / 2}\left|h^{\prime}\right|\left(M_{*}\right)^{1 / 2}\left(M_{*}^{\prime}\right)^{1 / 2} d \sigma d v_{*}, \\
T_{R}^{2} h(v)=2 M^{-1 / 2} \int_{\mathbb{R}^{N} \times \mathbb{S}^{N-1}} \mathbf{1}_{\left\{\left|v-v_{*}^{\prime}\right| \geq R / \sqrt{2}\right\}}\left(M^{\prime}\right)^{1 / 2}\left|h^{\prime}\right|\left(M_{*}\right)^{1 / 2}\left(M_{*}^{\prime}\right)^{1 / 2} d \sigma d v_{*} .
\end{array}\right.
$$

Now we follow the computations by Grad [19, Sections 2 and 3] (recalled in [13, Chapter 7, Section 2]) to compute and bound from above the kernel of these operators: we make the changes the variables

- $\sigma \in \mathbb{S}^{N-1}, v_{*} \in \mathbb{R}^{N} \longrightarrow \omega=\left(v^{\prime}-v\right) /\left|v^{\prime}-v\right| \in \mathbb{S}^{N-1}, v_{*} \in \mathbb{R}^{N}$ (the jacobian is bounded by a constant);

- then $\omega \in \mathbb{S}^{N-1}, v_{*} \in \mathbb{R}^{N} \longrightarrow \omega \in \mathbb{S}^{N-1}, u=v-v_{*} \in \mathbb{R}^{N}$ (the jacobian is equal to 1);

- then keeping $\omega$ fixed, decompose orthogonally $u=u_{0} \omega+W$ with $u_{0} \in \mathbb{R}$ and $W \in \omega^{\perp}$ (the jacobian is equal to 1 );

- finally keeping $W \in V^{\perp}$ fixed, $\omega \in \mathbb{S}^{N-1}, u_{0} \in \mathbb{R} \longrightarrow V=u_{0} \omega \in \mathbb{R}^{N}$ (the jacobian is $\left.(1 / 2)|V|^{-(N-1)}\right)$.

We get thus

$$
\begin{gathered}
\left|T_{R}^{1} h(v)\right| \leq C M(v)^{-1 / 2} \int_{V \in \mathbb{R}^{N}} \int_{W \in V^{\perp}} \\
|h(v+V)| M(v+V)^{1 / 2} \mathbf{1}_{\{|V| \geq R / \sqrt{2}\}}|V|^{-(N-1)} M(v+W)^{1 / 2} M(v+V+W)^{1 / 2} d V d W .
\end{gathered}
$$

Then using that ( $\omega$ denotes the unit vector of $V$ )

$$
\begin{aligned}
M(v+W)^{1 / 2} M(v+V+W)^{1 / 2}=M(V)^{1 / 4} M(V+2(W+v))^{1 / 4} & \\
& \leq M(V)^{1 / 4} M(W+(v-(v \cdot \omega) \omega)),
\end{aligned}
$$


we obtain the bound from above

$$
\left|T_{R}^{1} h(v)\right| \leq C M(v)^{-1 / 2} \int_{\mathbb{R}^{N}}|h(v+V)| M(v+V)^{1 / 2} \mathbf{1}_{\{|V| \geq R / \sqrt{2}\}}|V|^{-(N-1)} e^{-\frac{1}{4}|V|^{2}} d V .
$$

By Young's inequality one deduces immediately the convergence to 0 of $T_{R}^{1}$ in the operator norm with explicit rate. On the other hand for $T_{R}^{2}$ we use first that

$$
\left\|\left|\mathbf{1}_{\{|\cdot| \geq r\}} T_{R}^{2}\right|\right\|_{L^{2}(M)} \leq \||| \mathbf{1}_{\{|\cdot| \geq r\}} T_{R}||_{L^{2}(M)} \leq C(1+r)^{-1 / 2}
$$

with explicit constant by Grad [19, Section 4] (or see [13, Chapter 7, Section 2] again). Thus we pick $\varepsilon>0$ and then $r$ such that

$$
\|\left.\left|\mathbf{1}_{\{|\cdot| \geq r\}} T_{R}^{2}\right|\right|_{L^{2}(M)} \leq \varepsilon / 2 .
$$

Then using again the changes of variables detailed above we get

$$
\begin{aligned}
\left|\mathbf{1}_{\{|v| \leq r\}} T_{R}^{2} h(v)\right| \leq C M(v)^{-1 / 2} \mathbf{1}_{\{|v| \leq r\}} \int_{\mathbb{R}^{N}}|h(v+V)| M(v+V)^{1 / 2}|V|^{-(N-1)} & \\
& {\left[\int_{V^{\perp}} M(v+W)^{1 / 2} M(v+V+W)^{1 / 2} \mathbf{1}_{\{|W| \geq R / \sqrt{2}\}} d W\right] d V . }
\end{aligned}
$$

We use that

$$
M(v+W)^{1 / 4} M(v+V+W)^{1 / 4} \leq M(v+W)^{1 / 4} \leq M(v)^{-1 / 4} M(W)^{1 / 8}
$$

and

$$
M(v+W)^{1 / 4} M(v+V+W)^{1 / 4} \leq M(V)^{1 / 8}
$$

to obtain

$$
\begin{aligned}
& \left|\mathbf{1}_{\{|v| \leq r\}} T_{R}^{2} h(v)\right| \leq C e^{3 r^{2} / 4} \\
& \int_{\mathbb{R}^{N}}|h(v+V)| M(v+V)^{1 / 2}|V|^{-N-1} M(V)^{1 / 8}\left[\int_{V^{\perp}} M(W)^{1 / 8} \mathbf{1}_{\{|W| \geq R / \sqrt{2}\}} d W\right] d V .
\end{aligned}
$$

Since the function $|V|^{-N-1} M(V)^{1 / 8}$ belongs to $L^{1}$, the convolution according to this function is bounded from $L^{2}$ into $L^{2}$, and we deduce that

$$
\left\|\mathbf{1}_{\{|\cdot| \leq r\}} T_{R}^{2}\right\|_{L^{2}(M)} \leq C_{r}\left[\int_{\mathbb{R}^{N-1}} M(W)^{1 / 8} \mathbf{1}_{\{|W| \geq R / \sqrt{2}\}} d W\right]\|h\|_{L^{2}(M)}
$$

and thus, for $R$ big enough,

$$
\|\left.\left|\mathbf{1}_{\{|| \mid \leq r\}} T_{R}^{2}\right|\right|_{L^{2}(M)} \leq \varepsilon / 2 .
$$

Together with (2.1) this shows that $T_{R}^{2}$ goes to 0 in the operator norm with explicit rate, which ends the proof. 
Step 2. Let us do a dyadic decomposition of $D^{\mathcal{B}}(h)$. We fix a parameter $R>1$ and we use the following decomposition of identity:

$$
\mathbf{1}=\mathbf{1}_{\{|u| \leq R\}}+\sum_{n \geq 1} \mathbf{1}_{\left\{R^{n} \leq|u| \leq R^{n+1}\right\}}
$$

to obtain

$$
D^{\mathcal{B}}(h) \geq C \sum_{n \geq 0} R^{(n+1) \gamma} \tilde{D}_{n}^{\mathcal{B}}(h)
$$

with

$$
\tilde{D}_{n}^{\mathcal{B}}(h)=\int_{\mathbb{R}^{N} \times \mathbb{R}^{N} \times \mathbb{S}^{N-1}} \mathbf{1}_{\left\{R^{n} \leq\left|v-v_{*}\right| \leq R^{n+1}\right\}}\left[h_{*}^{\prime}+h^{\prime}-h_{*}-h\right]^{2} M M_{*} d v d v_{*} d \sigma
$$

for $n \geq 1$, and

$$
\tilde{D}_{0}^{\mathcal{B}}(h)=\int_{\mathbb{R}^{N} \times \mathbb{R}^{N} \times \mathbb{S}^{N-1}} \mathbf{1}_{\left\{\left|v-v_{*}\right| \leq R\right\}}\left[h_{*}^{\prime}+h^{\prime}-h_{*}-h\right]^{2} M M_{*} d v d v_{*} d \sigma .
$$

Now if we define

$$
D_{k}^{\mathcal{B}}(h)=\int_{\mathbb{R}^{N} \times \mathbb{R}^{N} \times \mathbb{S}^{N-1}} \mathbf{1}_{\left\{\left|v-v_{*}\right| \leq R^{k+1}\right\}}\left[h_{*}^{\prime}+h^{\prime}-h_{*}-h\right]^{2} M M_{*} d v d v_{*} d \sigma
$$

for any $k \geq 0$, we have

$$
\begin{aligned}
\sum_{k \geq 0} R^{(k+1) \gamma} D_{k}^{\mathcal{B}}(h)=\sum_{k \geq 0} R^{(k+1) \gamma} & \sum_{0 \leq n \leq k} \tilde{D}_{n}^{\mathcal{B}}(h) \\
= & \sum_{n \geq 0} \tilde{D}_{n}^{\mathcal{B}}(h)\left(\sum_{k \geq n} R^{(k+1) \gamma}\right)=S \sum_{n \geq 0} R^{(n+1) \gamma} \tilde{D}_{n}^{\mathcal{B}}(h)
\end{aligned}
$$

where the constant

$$
S=\sum_{k \geq 0}\left(R^{\gamma}\right)^{k}
$$

is finite thanks to the fact that $R>1$. Thus we deduce that

$$
D^{\mathcal{B}}(h) \geq \frac{C}{S} \sum_{n \geq 0} R^{(n+1) \gamma} D_{n}^{\mathcal{B}}(h)
$$

Step 3. In this step we estimate each term of the dyadic decomposition. We fix $n_{0} \in \mathbb{N}$ (to be latter chosen big enough) and we estimate $D_{n}^{\mathcal{B}}(h)$ for $n \geq n_{0}$. We denote $\chi_{r}$ the indicator function depending on the four variables $v, v_{*}, v^{\prime}, v_{*}^{\prime}$ such that at least one of these four points belongs to $B(0, r)$. We also define the shorthand

$$
\Delta(F)=\left[F^{\prime}+F_{*}^{\prime}-F_{*}-F\right] .
$$


Then

$$
\begin{aligned}
D_{n}^{\mathcal{B}}(h)=\int_{\mathbb{R}^{N} \times \mathbb{R}^{N} \times \mathbb{S}^{N-1}} \mathbf{1}_{\left\{\left|v-v_{*}\right| \leq R^{n+1}\right\}} \Delta(h)^{2} M M_{*} d v d v_{*} d \sigma \\
\quad \geq \int_{\mathbb{R}^{N} \times \mathbb{R}^{N} \times \mathbb{S}^{N-1}} \mathbf{1}_{\left\{\left|v-v_{*}\right| \leq R^{n+1}\right\}} \chi_{r}\left(v, v_{*}, v^{\prime}, v_{*}^{\prime}\right) \Delta(h)^{2} M M_{*} d v d v_{*} d \sigma .
\end{aligned}
$$

We take $r=R^{n+2}-R^{n+1}$ and we denote $h_{k}=h \mathbf{1}_{\left\{|\cdot| \leq R^{k}\right\}}$. If one of the four collision points belongs to $B\left(0, R^{n+2}-R^{n+1}\right)$ and the relative velocity is bounded by $R^{n+1}$, the collision sphere is included in $B\left(0, R^{n+2}\right)$. Thus we deduce

$$
D_{n}^{\mathcal{B}}(h) \geq \int_{\mathbb{R}^{N} \times \mathbb{R}^{N} \times \mathbb{S}^{N-1}} \mathbf{1}_{\left\{\left|v-v_{*}\right| \leq R^{n+1}\right\}} \chi_{r}\left(v, v_{*}, v^{\prime}, v_{*}^{\prime}\right) \Delta\left(h_{n+2}\right)^{2} M M_{*} d v d v_{*} d \sigma .
$$

Now we remove the indicator function $\chi_{r}$ by bounding from above the term corresponding to $1-\chi_{r}$, that is when all the four collision points have a modulus greater than $R^{n+2}-R^{n+1}$. Simple computations yield

$$
\begin{aligned}
D_{n}^{\mathcal{B}}(h) \geq \int_{\mathbb{R}^{N} \times \mathbb{R}^{N} \times \mathbb{S}^{N-1}} \mathbf{1}_{\left\{\left|v-v_{*}\right| \leq R^{n+1}\right\}} \Delta\left(h_{n+2}\right)^{2} M & M_{*} d v d v_{*} d \sigma \\
& -C_{1} e^{-\left(R^{n+2}-R^{n+1}\right)}\left\|h_{n+2}\right\|_{L^{2}(M)}^{2}
\end{aligned}
$$

for an explicit constant $C_{1}>0$. Then

$$
\begin{gathered}
\int_{\mathbb{R}^{N} \times \mathbb{R}^{N} \times \mathbb{S}^{N-1}} \mathbf{1}_{\left\{\left|v-v_{*}\right| \leq R^{n+1}\right\}} \Delta\left(h_{n+2}\right)^{2} M M_{*} d v d v_{*} d \sigma \\
=-4 \int_{\mathbb{R}^{N} \times \mathbb{R}^{N} \times \mathbb{S}^{N-1}} \mathbf{1}_{\left\{\left|v-v_{*}\right| \leq R^{n+1}\right\}} h_{n+2}\left[\left(h_{n+2}\right)^{\prime}+\left(h_{n+2}\right)_{*}^{\prime}-\left(h_{n+2}\right)_{*}\right] M M_{*} d v d v_{*} d \sigma \\
+4 \int_{\mathbb{R}^{N} \times \mathbb{R}^{N} \times \mathbb{S}^{N-1}} \mathbf{1}_{\left\{\left|v-v_{*}\right| \leq R^{n+1}\right\}} h_{n+2}^{2} M M_{*} d v d v_{*} d \sigma \\
\geq-4 \int_{\mathbb{R}^{N} \times \mathbb{R}^{N} \times \mathbb{S}^{N-1}} h_{n+2}\left[\left(h_{n+2}\right)^{\prime}+\left(h_{n+2}\right)_{*}^{\prime}-\left(h_{n+2}\right)_{*}\right] M M_{*} d v d v_{*} d \sigma \\
-4 \int_{\mathbb{R}^{N}}\left(K_{R^{n+1}}^{\mathcal{B}} h_{n+2}\right) h_{n+2} M d v \\
+4 \int_{\mathbb{R}^{N} \times \mathbb{R}^{N} \times \mathbb{S}^{N-1}} \mathbf{1}_{\left\{\left|v-v_{*}\right| \leq R^{n+1}\right\}} h_{n}^{2} M M_{*} d v d v_{*} d \sigma
\end{gathered}
$$


and thus we deduce that

$$
\begin{gathered}
\int_{\mathbb{R}^{N} \times \mathbb{R}^{N} \times \mathbb{S}^{N-1}} \mathbf{1}_{\left\{\left|v-v_{*}\right| \leq R^{n+1}\right\}} \Delta\left(h_{n+2}\right)^{2} M M_{*} d v d v_{*} d \sigma \\
\geq-4 \int_{\mathbb{R}^{N} \times \mathbb{R}^{N} \times \mathbb{S}^{N-1}} h_{n+2}\left[\left(h_{n+2}\right)^{\prime}+\left(h_{n+2}\right)_{*}^{\prime}-\left(h_{n+2}\right)_{*}\right] M M_{*} d v d v_{*} d \sigma \\
+4 \int_{\mathbb{R}^{N} \times \mathbb{R}^{N} \times \mathbb{S}^{N-1}} h_{n}^{2} M M_{*} d \sigma d v_{*} d v \\
-4 \int_{\mathbb{R}^{N}}\left(K_{R^{n+1}}^{\mathcal{B}} h_{n+2}\right) h_{n+2} M d v \\
-4 \int_{\mathbb{R}^{N} \times \mathbb{R}^{N} \times \mathbb{S}^{N-1}} \mathbf{1}_{\left\{\left|v-v_{*}\right| \geq R^{n+1}\right\}} h_{n}^{2} M M_{*} d v d v_{*} d \sigma .
\end{gathered}
$$

From Lemma 2.1 we have

$$
-4 \int_{\mathbb{R}^{N}}\left(K_{R^{n+1}}^{\mathcal{B}} h_{n+2}\right) h_{n+2} M d v \geq-\epsilon_{1}\left(R^{n+1}\right)\left\|h_{n+2}\right\|_{L^{2}(M)}^{2}
$$

where $\epsilon_{1}(r)$ is an explicit function going to 0 as $r$ goes to infinity. Also when $v \in B\left(0, R^{n}\right)$ and $\left|v-v_{*}\right| \geq R^{n+1}$ we have by triangular inequality $\left|v_{*}\right| \geq R^{n+1}-R^{n}$, and thus simple computations show that

$$
4 \int_{\mathbb{R}^{N} \times \mathbb{R}^{N} \times \mathbb{S}^{N-1}} \mathbf{1}_{\left\{\left|v-v_{*}\right| \geq R^{n+1}\right\}} h_{n}^{2} M M_{*} d v d v_{*} d \sigma \leq C_{2} e^{-\left(R^{n+1}-R^{n}\right)}\left\|h_{n+2}\right\|_{L^{2}(M)}^{2} .
$$

Collecting every terms we deduce

$$
\begin{aligned}
& \sum_{n \geq n_{0}} R^{(n+1) \gamma} D_{n}^{\mathcal{B}}(h) \geq \\
& \sum_{n \geq n_{0}} R^{(n+1) \gamma}\left[-4 \int_{\mathbb{R}^{N} \times \mathbb{R}^{N} \times \mathbb{S}^{N-1}} h_{n+2}\left[\left(h_{n+2}\right)^{\prime}+\left(h_{n+2}\right)_{*}^{\prime}-\left(h_{n+2}\right)_{*}\right] M M_{*} d v d v_{*} d \sigma\right. \\
& \quad+4 R^{2 \gamma} \int_{\mathbb{R}^{N} \times \mathbb{R}^{N} \times \mathbb{S}^{N-1}} h_{n+2}^{2} M M_{*} d v d v_{*} d \sigma \\
& \quad-C_{1} e^{-\left(R^{n+2}-R^{n+1}\right)}\left\|h_{n+2}\right\|_{L^{2}(M)}^{2}-\epsilon_{1}\left(R^{n+1}\right)\left\|h_{n+2}\right\|_{L^{2}(M)}^{2} \\
& \\
& \left.\quad-C_{2} e^{-\left(R^{n+1}-R^{n}\right)}\left\|h_{n+2}\right\|_{L^{2}(M)}^{2}\right]
\end{aligned}
$$


which writes

$$
\begin{gathered}
\sum_{n \geq n_{0}} R^{(n+1) \gamma} D_{n}^{\mathcal{B}}(h) \geq \sum_{n \geq n_{0}} R^{(n+1) \gamma}\left[\int_{\mathbb{R}^{N} \times \mathbb{R}^{N} \times \mathbb{S}^{N-1}} \Delta\left(h_{n+2}\right)^{2} M M_{*} d v d v_{*} d \sigma\right. \\
-4\left(1-R^{2 \gamma}\right)\left\|h_{n+2}\right\|_{L^{2}(M)}^{2}-C_{1} e^{-\left(R^{n+2}-R^{n+1}\right)}\left\|h_{n+2}\right\|_{L^{2}(M)}^{2} \\
\left.\quad-\epsilon_{1}\left(R^{n+1}\right)\left\|h_{n+2}\right\|_{L^{2}(M)}^{2}-C_{2} e^{-\left(R^{n+1}-R^{n}\right)}\left\|h_{n+2}\right\|_{L^{2}(M)}^{2}\right] .
\end{gathered}
$$

Now we use the explicit spectral gap for Maxwell molecules to get

$$
\int_{\mathbb{R}^{N} \times \mathbb{R}^{N} \times \mathbb{S}^{N-1}} \Delta\left(h_{n+2}\right)^{2} M M_{*} d v d v_{*} d \sigma \geq \lambda\left\|h_{n+2}-\Pi\left(h_{n+2}\right)\right\|_{L^{2}(M)}^{2}
$$

for an explicit $\lambda>0$. Hence we deduce that

$$
\begin{gathered}
\sum_{n \geq n_{0}} R^{(n+1) \gamma} D_{n}^{\mathcal{B}}(h) \geq \sum_{n \geq n_{0}} R^{(n+1) \gamma}\left[\lambda\left\|h_{n+2}\right\|_{L^{2}(M)}^{2}-\lambda\left\|\Pi\left(h_{n+2}\right)\right\|_{L^{2}(M)}^{2}\right. \\
-4\left(1-R^{2 \gamma}\right)\left\|h_{n+2}\right\|_{L^{2}(M)}^{2}-C_{1} e^{-\left(R^{n+2}-R^{n+1}\right)}\left\|h_{n+2}\right\|_{L^{2}(M)}^{2} \\
\left.\quad-\epsilon_{1}\left(R^{n+1}\right)\left\|h_{n+2}\right\|_{L^{2}(M)}^{2}-C_{2} e^{R^{n+1}-R^{n}}\left\|h_{n+2}\right\|_{L^{2}(M)}^{2}\right] .
\end{gathered}
$$

Since $\Pi(h)=0$, we have

$$
\begin{gathered}
\left\|\Pi\left(h_{n+2}\right)\right\|^{2}=\left\|\Pi\left(h \mathbf{1}_{\left\{|\cdot| \geq R^{n+2}\right\}}\right)\right\|^{2} \leq\left(\int_{\left\{|v| \geq R^{n+2}\right\}}|v|^{2+|\gamma|} M(v) d v\right)\left\|h\langle v\rangle^{\gamma / 2}\right\|_{L^{2}(M)}^{2} \\
\leq C_{3} e^{-R^{n+2}}\left\|h\langle v\rangle^{\gamma / 2}\right\|_{L^{2}(M)}^{2}
\end{gathered}
$$

Now if we choose $R-1>0$ small enough such that

$$
4\left(1-R^{2 \gamma}\right) \leq \frac{\lambda}{8}
$$

then $n_{0}$ big enough so that $R^{n+2}-R^{n+1}=R^{n+1}(R-1)$ and $R^{n+1}-R^{n}=R^{n}(R-1)$ big enough such that

$$
\forall n \geq n_{0}, \quad C_{1} e^{-\left(R^{n+2}-R^{n+1}\right)}, C_{2} e^{-\left(R^{n+1}-R^{n}\right)} \leq \frac{\lambda}{8}
$$

and also $n_{0}$ big enough such that $R^{n+1}$ big enough such that

$$
\forall n \geq n_{0}, \quad \epsilon_{1}\left(R^{n+1}\right) \leq \frac{\lambda}{8}
$$


we obtain for this choice of $n_{0}$ and $R$ :

$$
\begin{gathered}
\sum_{n \geq n_{0}} R^{(n+1) \gamma} D_{n}^{\mathcal{B}}(h) \geq \frac{\lambda}{2} \sum_{n \geq n_{0}} R^{(n+1) \gamma}\left\|h_{n+2}\right\|_{L^{2}(M)}^{2}-C_{3} \lambda\left(\sum_{n \geq n_{0}} e^{-R^{n+2}}\right)\left\|h\langle v\rangle^{\gamma / 2}\right\|_{L^{2}(M)}^{2} \\
\geq\left[C_{4} R^{n_{0} \gamma}-C_{5} e^{-R^{n_{0}}}\right]\left\|h\langle v\rangle^{\gamma / 2}\right\|_{L^{2}(M)}^{2}
\end{gathered}
$$

for some explicit constants $C_{4}, C_{5}>0$ independent on $n_{0}$. Thus by taking $n_{0}$ large enough we deduce that

$$
\sum_{n \geq n_{0}} R^{(n+1) \gamma} D_{n}^{\mathcal{B}}(h) \geq C_{6}\left\|h\langle v\rangle^{\gamma / 2}\right\|^{2}
$$

for some explicit constant $C_{6}>0$. Coming back to $D^{\mathcal{B}}(h)$, this ends the proof of Theorem 1.1] in the case $\gamma<0$.

2.3. Regularity for long-range interactions. We suppose here that the collision kernel $B$ satisfies (1.1), (1.2), (1.4), with $\alpha>0$ (the case $\alpha=0$ is deduced from Theorem 1.11). Thus we are reduced to the case where $B\left(\left|v-v_{*}\right|, \cos \theta\right)=$ $\left|v-v_{*}\right|^{\gamma} \theta^{-(N-1)-\alpha}$. By symmetrizing the Dirichlet form with the change of variable $\sigma \rightarrow-\sigma$, we can finally reduce to the case where $B\left(\left|v-v_{*}\right|, \cos \theta\right)=\mid v-$ $\left.v_{*}\right|^{\gamma} \theta^{-(N-1)-\alpha} \mathbf{1}_{\theta \in[0, \pi / 2]}$. We pick $h \in L^{2}(M)$ orthogonal to the null space of $L^{\mathcal{B}}$.

We start by restricting the velocity variables to a bounded domain. Let us fix $R>0$, and let us denote by $\mathcal{I}_{R}$ a $C^{\infty}$ mollified indicator function of the variables $v, v_{*}$ which is 1 on $B_{R}=B(0, R)$ and 0 outside $B(0, R+1)$.

We control from below the Dirichlet form by

$$
D^{\mathcal{B}}(h) \geq \frac{1}{4} \int_{\mathbb{R}^{N} \times \mathbb{R}^{N} \times \mathbb{S}^{N-1}} B \mathcal{I}_{R}\left[h^{\prime}+h_{*}^{\prime}-h-h_{*}\right]^{2} M M_{*} d v d v_{*} d \sigma
$$

and we develop it as

$$
\begin{aligned}
D^{\mathcal{B}} \geq \frac{1}{4} \int_{\mathbb{R}^{N} \times \mathbb{R}^{N} \times \mathbb{S}^{N-1}} B \mathcal{I}_{R}\left(\left[h^{\prime}-h\right]^{2}+\left[h_{*}^{\prime}-h_{*}\right]^{2}\right) M M_{*} d v d v_{*} d \sigma \\
+\frac{1}{2} \int_{\mathbb{R}^{N} \times \mathbb{R}^{N} \times \mathbb{S}^{N-1}} B \mathcal{I}_{R}\left(h^{\prime}-h\right)\left(h_{*}^{\prime}-h_{*}\right) M M_{*} d v d v_{*} d \sigma
\end{aligned}
$$

The pre-postcollisional change of variable on the second term and the change of variable $\left(v, v_{*}, \sigma\right) \rightarrow\left(v_{*}, v,-\sigma\right)$ on the first term yield

$$
\begin{aligned}
D^{\mathcal{B}} \geq \frac{1}{2} \int_{\mathbb{R}^{N} \times \mathbb{R}^{N} \times \mathbb{S}^{N-1}} B \mathcal{I}_{R}\left(h^{\prime}-h\right)^{2} M M_{*} d v d v_{*} d \sigma \\
-\int_{\mathbb{R}^{N} \times \mathbb{R}^{N} \times \mathbb{S}^{N-1}} B \mathcal{I}_{R} h_{*}\left(h^{\prime}-h\right) M M_{*} d v d v_{*} d \sigma=: I_{1}^{R}+I_{2}^{R} .
\end{aligned}
$$


Now we estimate separately $I_{1}^{R}$ from below and $I_{2}^{R}$ from above. For the term $I_{1}^{R}$, the Carleman representation (see [11]) yields

$$
I_{1}^{R} \geq C \int_{B_{R} \times B_{R}} S\left(v, v^{\prime}\right) \frac{\left(h^{\prime}-h\right)^{2}}{\left|v-v^{\prime}\right|^{N+\alpha}} d v d v^{\prime}
$$

where

$$
S\left(v, v^{\prime}\right)=M(v) \int_{E_{v, v^{\prime}} \cap B_{R}} \mathbf{1}_{B_{R}}\left(v_{*}\right)\left|v^{\prime}-v_{*}^{\prime}\right|^{1+\gamma+\alpha} \mathbf{1}_{\left\{\left|v^{\prime}-v\right| \leq\left|v_{*}^{\prime}-v\right|\right\}} M_{*}^{\prime} d v_{*}^{\prime}
$$

and $E_{v, v^{\prime}}$ is the hyperplan containing $v$ and orthogonal to $v-v^{\prime}$ (for the derivation of this formula, see [30, Section 4]). The second indicator function in the formula for $S\left(v, v^{\prime}\right)$ comes from the restriction to $\theta \in[0, \pi / 2]$ by the symmetrization above. It is easily seen that $S\left(v, v^{\prime}\right)$ is bounded from below by some constant $C>0$ on $B_{R} \times B_{R}$. It follows that

$$
I_{1}^{R} \geq C \int_{B_{R} \times B_{R}} \frac{\left(h^{\prime}-h\right)^{2}}{\left|v-v^{\prime}\right|^{N+\alpha}} d v d v^{\prime} \geq C_{1}\|h\|_{H^{\alpha / 2}\left(B_{R}\right)}^{2}
$$

for some constant $C_{1}>0$ (for the last inequality see for instance [1]).

As for the second term $I_{2}^{R}$, we use the change of variable of the cancellation lemma in [4, Section 3]: keeping $v_{*}$ fixed, change $v, \sigma$ into $v^{\prime}, \sigma$ (the jacobian is $\cos ^{-N} \theta / 2$ ). We obtain

$$
\begin{aligned}
I_{2}^{R}= & \int_{\mathbb{R}^{N} \times \mathbb{R}^{N} \times \mathbb{S}^{N-1}} B h_{*} h\left|v-v_{*}\right|^{\gamma} M_{*} \\
& \quad b(\cos \theta)\left[M\left(\psi_{\sigma}(v)\right) \cos ^{-N-\gamma} \theta / 2 \mathcal{I}_{R}\left(\psi_{\sigma}(v), v_{*}\right)-M(v) \mathcal{I}_{R}\left(v, v_{*}\right)\right] d v d v_{*} d \sigma
\end{aligned}
$$

where $\psi_{\sigma}(v)$ is the transformation introduced in [4, Lemma 1]: it is the point in the plan defined by $v, v_{*}, \sigma$ such that

$$
\left(\psi_{\sigma}(v)-v\right) \perp\left(v_{*}-v\right) \text { and }\left(\psi_{\sigma}(v)-v_{*}\right) \cdot\left(v-v_{*}\right)=\cos \theta / 2 .
$$

Now let us fix $v, v_{*}$ and $\theta$. Then the modulus $\left|\psi_{\sigma}(v)-v\right|=\tan \theta / 2\left|v-v_{*}\right|$ is fixed, and the vector $\psi_{\sigma}(v)-v$ satisfies

$$
\psi_{\sigma}(v)-v=\left|\psi_{\sigma}(v)-v\right| \omega
$$

where $\omega$ is the opposite of the unit vector supporting the projection of $\sigma$ on the plan orthogonal to $v-v_{*}$ (see [4, Figure 1]). It motivates the study of quantities like

$$
I(\varphi)=\int_{\mathbb{S}_{v-v_{*}}^{N-2}}(\varphi(v+\rho \omega)-\varphi(v)) d \omega
$$


where $\varphi$ denotes some $C^{2}$ function on $\mathbb{R}^{N}, \mathbb{S}_{v-v_{*}}^{N-2}$ denotes the unit sphere in the plan orthogonal to $v-v_{*}$, and $\rho>0$. If $\nabla \varphi$ denotes the gradient of $\varphi$ and $\nabla^{2} \varphi$ denotes its Hessian matrix, one has the following Taylor expansion:

$$
\varphi(v+\rho \omega)=\varphi(v)+\rho(\nabla \varphi(v) \cdot \omega)+\frac{\rho^{2}}{2}\left\langle\nabla^{2} \varphi\left(v+\rho^{\prime} \omega\right) \cdot \omega, \omega\right\rangle
$$

for some $0 \leq \rho^{\prime} \leq \rho$. By bounding the last term and taking the integral over $\mathbb{S}_{v-v_{*}}^{N-2}$, we get the estimate

$$
\left|I(\varphi)-\rho\left(\int_{\mathbb{S}_{v-v_{*}^{\prime}}^{N-2}} d \sigma(\nabla \varphi(v) \cdot \sigma)\right)\right| \leq \frac{\rho^{2}}{2}\left|\mathbb{S}^{N-2}\right|\|\varphi\|_{W^{2, \infty}} .
$$

As the term involving $\nabla \varphi$ vanishes by symmetry, we obtain

$$
|I(\varphi)| \leq \frac{\rho^{2}}{2}\left|\mathbb{S}^{N-2}\right|\|\varphi\|_{W^{2, \infty}}
$$

We apply this computation to $\varphi(v)=M(v) \mathcal{I}\left(v, v_{*}\right)$ with $\rho=\left|\psi_{\sigma}(v)-v\right|=$ $\tan \theta / 2\left|v-v_{*}\right|$ to find

$$
\begin{aligned}
& \forall v, v_{*} \in B_{R}, \mid \int_{\mathbb{S}^{N-1}} b(\cos \theta)[\left.M\left(\psi_{\sigma}(v)\right) \mathcal{I}\left(\psi_{\sigma}(v), v_{*}\right)-M(v) \mathcal{I}\left(v, v_{*}\right)\right] d \sigma \mid \\
& \leq C \int_{0}^{\pi / 2} \theta^{-(N-1)-\alpha} \tan ^{2} \theta / 2 \sin ^{N-2} \theta / 2 d \theta \leq C_{2}
\end{aligned}
$$

for some finite constant $C_{2}>0$. Finally we have immediately

$$
\begin{aligned}
& \int_{\mathbb{S}^{N-1}} b(\cos \theta)\left|\cos ^{-N-\gamma} \theta / 2-1\right| d \sigma \\
& \leq \int_{0}^{\pi / 2} \theta^{-(N-1)-\alpha}\left|\cos ^{-N-\gamma} \theta / 2-1\right| \sin ^{N-2} \theta / 2 d \theta \leq C_{3}
\end{aligned}
$$

for some finite constant $C_{3}>0$. We thus deduce that

$$
\left|I_{2}^{R}\right| \leq C\|h\|_{L^{2}\left(B_{R}\right)}^{2} \leq C_{4}\left\|h\langle v\rangle^{\gamma / 2}\right\|_{L^{2}(M)}^{2} .
$$

Now we can conclude the proof of Theorem 1.2. For any $R>0$, we have

$$
\|h\|_{H^{\alpha / 2}\left(B_{R}\right)}^{2} \leq C_{1}^{-1} I_{1}^{R} \leq C_{1}^{-1}\left[D^{\mathcal{B}}(h)+\left|I_{2}^{R}\right|\right] \leq C_{1}^{-1}\left[D^{\mathcal{B}}(h)+C_{4}\left\|h\langle v\rangle^{\gamma / 2}\right\|_{L^{2}(M)}^{2}\right] .
$$

Since $\Pi(h)=0$ we can use the coercivity estimate of Theorem 1.1

$$
\left\|h\langle v\rangle^{\gamma / 2}\right\|_{L^{2}(M)}^{2} \leq C_{5} D^{\mathcal{B}}(h)
$$

to deduce finally

$$
\|h\|_{H^{\alpha / 2}\left(B_{R}\right)}^{2} \leq C_{1}^{-1}\left[1+C_{4} C_{5}\right] D^{\mathcal{B}}(h)
$$


Since it is valid for any $R>0$, we obtain

$$
D^{\mathcal{B}}(h) \geq C\|h\|_{H_{\mathrm{loc}}^{\alpha / 2}}^{2}
$$

for some explicit constant $C>0$. This ends the proof of Theorem 1.2.

Remark: It is easy to see that in our proof the dependency on $R$ of the constant in the control of the $H^{\alpha / 2}\left(B_{R}\right)$ norm by the Dirichlet form can be made explicit and grows exponentially in terms of $R$. More details on this point will be discussed in the forthcoming work [27].

\section{The LiNEARIZED LANDAU OPERATOR}

Note that here on the contrary to [7] we are not able to perform a grazing collision limit in the coercivity estimates for the linearized Boltzmann operator. Thus we do not try to deduce results on the linearized Landau operator from the Boltzmann case, instead we work directly on this operator.

3.1. Hard potentials and Maxwell molecules. We consider $h \in L^{2}(M)$ orthogonal to the null space of $L^{\mathcal{L}}$, we assume that $\gamma \geq 0$ and, thanks to the assumption (1.2), we reduce to the case $\Phi(z)=z^{\gamma}$.

Classical computations, which can be found in [14, Section 2] for instance, show that the linearized Landau operator $L^{\mathcal{L}}$ decomposes as

$$
L^{\mathcal{L}}=K^{\mathcal{L}}-A^{\mathcal{L}}
$$

where $K^{\mathcal{L}}$ is a (compact) bounded operator (with explicit bound $C_{K}^{\mathcal{L}}$ ) and $A^{\mathcal{L}}$ is a diffusion operator whose Dirichlet form satisfies

$$
\int_{\mathbb{R}^{N}}\left(A^{\mathcal{L}} h\right) h M d v=\int_{\mathbb{R}^{N}}\left(\nabla_{v} h\right)^{t} \mathcal{M}(v)\left(\nabla_{v} h\right) M d v
$$

where the matrix $\mathcal{M}$ is symmetric definite positive with its smallest eigenvalue bounded from below by $C\langle v\rangle^{\gamma}$ for an explicit constant $C>0$ (see [14, Section 2, Propositions 2.3 and 2.4]). Thus we deduce that

$$
\int_{\mathbb{R}^{N}}\left(A^{\mathcal{L}} h\right) h M d v \geq C \int_{\mathbb{R}^{N}}\left|\nabla_{v} h\right|^{2}\langle v\rangle^{\gamma} M d v .
$$

First, we recall that, as noticed in [25], a simpler way to recover the coercivity result from [14, Section 3, Theorem 3.1] is to apply the Bakry-Emery criterion (see [33, Chapter 9, Section 2]), which implies that $M$ satisfies a Poincaré inequality with constant 2 , and thus (as $h$ has zero mean)

$$
\int_{\mathbb{R}^{N}}\left|\nabla_{v} h\right|^{2}\langle v\rangle^{\gamma} M d v \geq \int_{\mathbb{R}^{N}}\left|\nabla_{v} h\right|^{2} M d v \geq 2 \int_{\mathbb{R}^{N}} h^{2} M d v .
$$


Now we want to obtain a stronger coercivity estimate. Thus we apply Bakry-Emery criterion to the measure

$$
m(v)=\langle v\rangle^{\gamma} M(v)=\exp \left[-|v|^{2}+\frac{\gamma}{2} \ln \left(1+|v|^{2}\right)\right]=: \exp [-\phi(v)] .
$$

A straightforward computation shows that

$$
\nabla^{2} \phi \geq(2-\gamma) I d
$$

which implies, as $(2-\gamma) \geq 1$ thanks to the assumptions on $\gamma$, that $m$ satisfies a Poincaré inequality with constant 1 , and thus

$$
\int_{\mathbb{R}^{N}}\left|\nabla_{v} h\right|^{2}\langle v\rangle^{\gamma} M d v \geq \int_{\mathbb{R}^{N}}\left[h-\left(\int_{\mathbb{R}^{N}} h\langle v\rangle^{\gamma} M d v\right)\right]^{2}\langle v\rangle^{\gamma} M d v .
$$

Hence by developing

$$
\int_{\mathbb{R}^{N}}\left|\nabla_{v} h\right|^{2}\langle v\rangle^{\gamma} M d v \geq \int_{\mathbb{R}^{N}} h^{2}\langle v\rangle^{\gamma} M d v-\left(\int_{\mathbb{R}^{N}} h\langle v\rangle^{\gamma} M d v\right)^{2} .
$$

Now as

$$
\left(\int_{\mathbb{R}^{N}} h\langle v\rangle^{\gamma} M d v\right)^{2} \leq C_{1}\left(\int_{\mathbb{R}^{N}} h^{2} M d v\right)
$$

for some explicit constant $C_{1}$, we deduce by collecting every term that

$$
\int_{\mathbb{R}^{N}}\left(A^{\mathcal{L}} h\right) h M d v \geq C_{2}\|h\|_{H^{1}\left(\langle v\rangle^{\gamma} M\right)}^{2}-C_{3}\|h\|_{L^{2}(M)}^{2}
$$

for some explicit constants $C_{2}, C_{3}>0$.

Besides we have by [7, Theorem 1.2]

$$
-\int_{\mathbb{R}^{N}}\left(L^{\mathcal{L}} h\right) h M d v \geq C_{4}\|h\|_{L^{2}(M)}^{2}
$$

for an explicit constant $C_{4}>0$. Now let us write

$$
\begin{aligned}
& \|h\|_{H^{1}\left(\langle v\rangle^{\gamma} M\right)}^{2} \leq C_{2}^{-1} \int_{\mathbb{R}^{N}}\left(A^{\mathcal{L}} h\right) h M d v+C_{2}^{-1} C_{3}\|h\|_{L^{2}(M)}^{2} \\
& \leq C_{2}^{-1}\left[-\int_{\mathbb{R}^{N}}\left(L^{\mathcal{L}} h\right) h M d v+\int_{\mathbb{R}^{N}}\left(K^{\mathcal{L}} h\right) h M d v+C_{3}\|h\|_{L^{2}(M)}^{2}\right] \\
& \leq C_{2}^{-1}\left[D^{\mathcal{L}}(h)+\left(C_{K}^{\mathcal{L}}+C_{3}\right)\|h\|_{L^{2}(M)}^{2}\right] \leq C_{2}^{-1}\left[1+\left(C_{K}^{\mathcal{L}}+C_{3}\right) C_{4}^{-1}\right] D^{\mathcal{L}}(h)
\end{aligned}
$$

which concludes half of the proof of Theorem 1.3 when $\gamma \geq 0$. It remains to control the $L^{2}(M)$ norm with weight $1+\gamma / 2$. 
Let us denote $g=h M^{1 / 2}$. Then

$$
\begin{gathered}
\|h\|_{H^{1}\left(\langle v\rangle^{\gamma} M\right)}^{2} \geq \int_{\mathbb{R}^{N}}|\nabla h|^{2} M\langle v\rangle^{\gamma} d v=\int_{\mathbb{R}^{N}}\left|\nabla \frac{g}{M^{1 / 2}}\right|^{2} M\langle v\rangle^{\gamma} d v= \\
\int_{\mathbb{R}^{N}}|\nabla g|^{2}\langle v\rangle^{\gamma} d v+\int_{\mathbb{R}^{N}}|g|^{2} \frac{|v|^{2}}{4}\langle v\rangle^{\gamma} d v+\int_{\mathbb{R}^{N}} g \nabla g \cdot v\langle v\rangle^{\gamma} d v \\
=\int_{\mathbb{R}^{N}}|\nabla g|^{2}\langle v\rangle^{\gamma} d v+\int_{\mathbb{R}^{N}}|g|^{2} \frac{|v|^{2}}{4}\langle v\rangle^{\gamma} d v-\int_{\mathbb{R}^{N}}|g|^{2}\left|\nabla\left(v\langle v\rangle^{\gamma}\right)\right| d v \\
\geq\left\|h|v|^{1+\gamma / 2}\right\|_{L^{2}(M)}^{2}-C\left\|h\langle v\rangle^{\gamma}\right\|_{L^{2}(M)}^{2}
\end{gathered}
$$

which implies immediately, combined to the previous estimate, inequality (1.8) in Theorem 1.3 .

3.2. Soft potentials. We follow almost the same path as for the linearized Boltzmann operator. The starting point is the following coercivity estimate in the Maxwell case

$$
\begin{aligned}
\frac{1}{2} \int_{\mathbb{R}^{N} \times \mathbb{R}^{N}}\left|v-v_{*}\right|^{2}\left\|\mathbf{P}\left(v-v_{*}\right)\left[(\nabla h)-(\nabla h)_{*}\right]\right\|^{2} M M_{*} d v d v_{*} & \\
& \geq \lambda\left[\|h\|_{H^{1}(M)}^{2}+\|h\langle v\rangle\|_{L^{2}(M)}^{2}\right]
\end{aligned}
$$

for some explicit constant $\lambda>0$, which has been proved in the previous subsection.

We assume that $\gamma<0$ and we pick $h \in L^{2}(M)$ orthogonal to the null space of $L^{\mathcal{L}}$. Using the assumption (1.2) we reduce to the case $\Phi(z)=\min \left\{z^{\gamma}, 1\right\}$.

Step 1. We first prove a technical lemma on $K^{\mathcal{L}}$, in the case of Maxwell molecules. We define for $R>0$

$K_{R}^{\mathcal{L}} h(v)=-M(v)^{-1} \nabla_{v} \cdot\left(\int_{\mathbb{R}^{N}}\left(1-\Theta_{R}\left(v-v_{*}\right)\right)\left|v-v_{*}\right|^{2} \mathbf{P}\left(v-v_{*}\right)(\nabla h)_{*} M M_{*} d v_{*}\right)$

where $\Theta_{R}$ is a $C^{\infty}$ function on $\mathbb{R}^{N}$ such that $0 \leq \Theta_{R} \leq 1, \Theta_{R}=1$ on $B(0, R)$ and $\Theta_{R}=0$ outside $B(0, R+1)$. Then

Lemma 3.1. The bounded operator $K_{R}^{\mathcal{L}}$ satisfies

$$
\left\|\mid K_{R}^{\mathcal{L}}\right\|_{L^{2}(M)} \stackrel{R \rightarrow \infty}{\longrightarrow} 0
$$

with explicit rate.

Proof of Lemma 3.1. It amounts to a differentiation under the integral, an integration by part, and Young's inequality. 
Step 2. We do the same dyadic decomposition of $D^{\mathcal{L}}(h)$ as for the Boltzmann case, to obtain

$$
D^{\mathcal{L}}(h) \geq C \sum_{n \geq 0} R^{(n+1) \gamma} D_{n}^{\mathcal{L}}(h)
$$

for some constant $C>0$, with

$$
D_{n}^{\mathcal{L}}(h)=\int_{\mathbb{R}^{N} \times \mathbb{R}^{N}} \mathbf{1}_{\left\{\left|v-v_{*}\right| \leq R^{n+1}\right\}}\left|v-v_{*}\right|^{2}\left\|\mathbf{P}\left(v-v_{*}\right)\left[(\nabla h)-(\nabla h)_{*}\right]\right\|^{2} M M_{*} d v d v_{*}
$$

for any $n \geq 0$.

Step 3. In this step we estimate each term of the dyadic decomposition. We fix $n_{0} \geq 0$ (to be later chosen big enough) and we estimate $D_{n}^{\mathcal{L}}(h)$ for $n \geq n_{0}$. We denote $\chi_{r}$ the indicator function depending on $v, v_{*}$ such that at least on of these two points belongs to $B(0, r)$. We also define the shorthand

$$
\Delta(F)=\left\|\mathbf{P}\left(v-v_{*}\right)\left[(\nabla F)-(\nabla F)_{*}\right]\right\| .
$$

Then

$$
\begin{aligned}
D_{n}^{\mathcal{L}}(h)=\int_{\mathbb{R}^{N} \times \mathbb{R}^{N}} & 1_{\left\{\left|v-v_{*}\right| \leq R^{n+1}\right\}}\left|v-v_{*}\right|^{2} \Delta(h)^{2} M M_{*} d v d v_{*} \\
& \geq \int_{\mathbb{R}^{N} \times \mathbb{R}^{N}} \mathbf{1}_{\left\{\left|v-v_{*}\right| \leq R^{n+1}\right\}} \chi_{r}\left(v, v_{*}\right)\left|v-v_{*}\right|^{2} \Delta(h)^{2} M M_{*} d v d v_{*} .
\end{aligned}
$$

We take $r=R^{n+2}-R^{n+1}$ and we denote $h_{k}=h \bar{\Theta}_{R^{k}}^{\eta}$ where $\bar{\Theta}_{R}^{\eta}$ is defined by $0 \leq \bar{\Theta}_{R}^{\eta} \leq 1, \bar{\Theta}_{R}^{\eta}=1$ on $B(0, R), \Theta_{R}=0$ outside $B\left(0, R+\eta^{-1}\right)$ and $\left|\nabla \bar{\Theta}_{R}^{\eta}\right| \leq \eta$ (with $\eta>0$ to be later chosen small enough).

If $v$ or $v_{*}$ belongs to $B\left(0, R^{n+2}-R^{n+1}\right)$ and the relative velocity is bounded by $R^{n+1}$, both points belong to $B\left(0, R^{n+2}\right)$. Thus we deduce

$$
D_{n}^{\mathcal{L}}(h) \geq \int_{\mathbb{R}^{N} \times \mathbb{R}^{N}} \mathbf{1}_{\left\{\left|v-v_{*}\right| \leq R^{n+1}\right\}}\left|v-v_{*}\right|^{2} \chi_{r}\left(v, v_{*}\right) \Delta\left(h_{n+2}\right)^{2} M M_{*} d v d v_{*} .
$$

Now we remove the indicator function $\chi_{r}$ by bounding from above the term corresponding to $1-\chi_{r}$, that is when $v$ and $v_{*}$ have a modulus greater than $R^{n+2}-R^{n+1}$. Simple computations yield

$$
\begin{aligned}
D_{n}^{\mathcal{L}}(h) \geq \int_{\mathbb{R}^{N} \times \mathbb{R}^{N}} \mathbf{1}_{\left\{\left|v-v_{*}\right| \leq R^{n+1}\right\}}\left|v-v_{*}\right|^{2} \Delta\left(h_{n+2}\right)^{2} M M_{*} d v d v_{*} \\
-C_{1} R^{2(n+1)} e^{-\left(R^{n+2}-R^{n+1}\right)}\left\|h_{n+2}\right\|_{H^{1}(M)}^{2}
\end{aligned}
$$

for an explicit constant $C_{1}>0$. Then we focus on the main term

$$
\int_{\mathbb{R}^{N} \times \mathbb{R}^{N}} \mathbf{1}_{\left\{\left|v-v_{*}\right| \leq R^{n+1}\right\}}\left|v-v_{*}\right|^{2} \Delta\left(h_{n+2}\right)^{2} M M_{*} d v d v_{*}
$$


Since $\mathbf{1}_{\left\{\left|v-v_{*}\right| \leq R^{n+1}\right\}} \geq \Theta_{R^{n+1}-1}\left(v-v_{*}\right)$, we first bound it from below by

$$
\int_{\mathbb{R}^{N} \times \mathbb{R}^{N}} \Theta_{R^{n+1}-1}\left(v-v_{*}\right)\left|v-v_{*}\right|^{2} \Delta\left(h_{n+2}\right)^{2} M M_{*} d v d v_{*}
$$

Then we proceed as in the Boltzmann case:

$$
\begin{aligned}
& \int_{\mathbb{R}^{N} \times \mathbb{R}^{N}} \Theta_{R^{n+1}-1}\left(v-v_{*}\right)\left|v-v_{*}\right|^{2} \Delta\left(h_{n+2}\right)^{2} M M_{*} d v d v_{*} \\
& =-2 \int_{\mathbb{R}^{N} \times \mathbb{R}^{N}} \Theta_{R^{n+1}-1}\left(v-v_{*}\right)\left|v-v_{*}\right|^{2}\left[\mathbf{P}\left(v-v_{*}\right)\left(\nabla h_{n+2}\right)\right] \cdot\left[\mathbf{P}\left(v-v_{*}\right)\left(\nabla h_{n+2}\right)_{*}\right] M M_{*} d v d v_{*} \\
& \quad+2 \int_{\mathbb{R}^{N} \times \mathbb{R}^{N}} \Theta_{R^{n+1}-1}\left(v-v_{*}\right)\left|v-v_{*}\right|^{2}\left\|\mathbf{P}\left(v-v_{*}\right)\left(\nabla h_{n+2}\right)\right\|^{2} M M_{*} d v d v_{*} \\
& \geq-2 \int_{\mathbb{R}^{N} \times \mathbb{R}^{N}}\left|v-v_{*}\right|^{2}\left[\mathbf{P}\left(v-v_{*}\right)\left(\nabla h_{n+2}\right)\right] \cdot\left[\mathbf{P}\left(v-v_{*}\right)\left(\nabla h_{n+2}\right)_{*}\right] M M_{*} d v d v_{*} \\
& \quad-2 \int_{\mathbb{R}^{N}}\left(K_{R^{n+1}-1}^{\mathcal{L}} h_{n+2}\right) h_{n+2} M d v \\
& \quad+2 \int_{\mathbb{R}^{N} \times \mathbb{R}^{N}} \Theta_{R^{n+1}-1}\left(v-v_{*}\right)\left|v-v_{*}\right|^{2}\left\|\mathbf{P}\left(v-v_{*}\right)\left(\nabla h_{n+2}\right)\right\|^{2} M M_{*} d v d v_{*} .
\end{aligned}
$$

Then we use that

$$
\begin{gathered}
\left\|\mathbf{P}\left(v-v_{*}\right)\left(\nabla h_{n+2}\right)\right\|^{2}=\left\|\mathbf{P}\left(v-v_{*}\right)\left(\nabla\left(\bar{\Theta}_{R^{n+2}}^{\eta} h\right)\right)\right\|^{2} \\
\geq(1-\varepsilon)\left\|\mathbf{P}\left(v-v_{*}\right)\left(\bar{\Theta}_{R^{n+2}}^{\eta} \nabla h\right)\right\|^{2}-C(\varepsilon)\left\|\mathbf{P}\left(v-v_{*}\right)\left(h \nabla \bar{\Theta}_{R^{n+2}}^{\eta}\right)\right\|^{2} \\
\geq(1-\varepsilon)\left\|\mathbf{P}\left(v-v_{*}\right)\left(\bar{\Theta}_{R^{n}}^{\eta} \nabla h\right)\right\|^{2}-C(\varepsilon)\left\|\mathbf{P}\left(v-v_{*}\right)\left(h \nabla \bar{\Theta}_{R^{n+2}}^{\eta}\right)\right\|^{2} \\
\geq(1-2 \varepsilon)\left\|\mathbf{P}\left(v-v_{*}\right)\left(\nabla\left(\bar{\Theta}_{R^{n}}^{\eta} h\right)\right)\right\|^{2} \\
\quad-C(\varepsilon)\left\|\mathbf{P}\left(v-v_{*}\right)\left(h \nabla \bar{\Theta}_{R^{n}}^{\eta}\right)\right\|^{2}-C(\varepsilon)\left\|\mathbf{P}\left(v-v_{*}\right)\left(h \nabla \bar{\Theta}_{R^{n+2}}^{\eta}\right)\right\|^{2} \\
\geq(1-2 \varepsilon)\left\|\mathbf{P}\left(v-v_{*}\right)\left(\nabla h_{n}\right)\right\|^{2}-C_{2}(\varepsilon) \eta h_{n+3} \|^{2}
\end{gathered}
$$

since $R^{n+2}+\eta^{-1} \leq R^{n+3}$ if $n \geq n_{0}$ is big enough. 
Hence we deduce that

$$
\begin{aligned}
& \int_{\mathbb{R}^{N} \times \mathbb{R}^{N}} \Theta_{R^{n+1}-1}\left(v-v_{*}\right)\left|v-v_{*}\right|^{2} \Delta\left(h_{n+2}\right)^{2} M M_{*} d v_{*} d v \\
& \geq-2 \int_{\mathbb{R}^{N} \times \mathbb{R}^{N}}\left|v-v_{*}\right|^{2}\left[\mathbf{P}\left(v-v_{*}\right)\left(\nabla h_{n+2}\right)\right] \cdot\left[\mathbf{P}\left(v-v_{*}\right)\left(\nabla h_{n+2}\right)_{*}\right] M M_{*} d v d v_{*} \\
& \quad+2(1-2 \varepsilon) \int_{\mathbb{R}^{N} \times \mathbb{R}^{N}}\left|v-v_{*}\right|^{2}\left\|\mathbf{P}\left(v-v_{*}\right)\left(\nabla h_{n}\right)\right\|^{2} M M_{*} d v d v_{*} \\
& \quad-2 \int_{\mathbb{R}^{N}}\left(K_{R^{n+1}-1}^{\mathcal{L}} h_{n+2}\right) h_{n+2} M d v-C_{2}(\varepsilon) \eta\left\|h_{n+3}\langle v\rangle\right\|_{L^{2}(M)}^{2} \\
& \quad-2 \int_{\mathbb{R}^{N} \times \mathbb{R}^{N}} \mathbf{1}_{\left\{\left|v-v_{*}\right| \geq R^{n+1}-1\right\}}\left|v-v_{*}\right|^{2}\left\|\mathbf{P}\left(v-v_{*}\right)\left(\nabla h_{n}\right)\right\|^{2} M M_{*} d v d v_{*} .
\end{aligned}
$$

Now we use that (from Lemma 3.1)

$$
-2 \int_{\mathbb{R}^{N}}\left(K_{R^{n+1}-1}^{\mathcal{L}} h_{n+2}\right) h_{n+2} M d v \geq-\epsilon_{2}\left(R^{n+1}-1\right)\left\|h_{n+2}\right\|_{H^{1}(M)}^{2}
$$

where $\epsilon_{2}(r)$ is an explicit function going to 0 as $r$ goes to infinity. Also simple computations show that

$$
\begin{aligned}
2 \int_{\mathbb{R}^{N} \times \mathbb{R}^{N}} 1_{\left\{\left|v-v_{*}\right| \geq R^{n+1}-1\right\}}\left|v-v_{*}\right|^{2} \| \mathbf{P}\left(v-v_{*}\right) & \left(\nabla h_{n}\right) \|^{2} M M_{*} d v d v_{*} \\
& \leq C_{3} R^{2 n} e^{-\left(R^{n+1}-R^{n}\right)}\left\|h_{n+2}\right\|_{H^{1}(M)}^{2} .
\end{aligned}
$$

Collecting every term we deduce

$$
\begin{aligned}
& \sum_{n \geq n_{0}} R^{(n+1) \gamma} D_{n}^{\mathcal{L}}(h) \geq \sum_{n \geq n_{0}} R^{(n+1) \gamma} \\
& {\left[\begin{array}{l}
-2 \int_{\mathbb{R}^{N} \times \mathbb{R}^{N}}\left|v-v_{*}\right|^{2}\left[\mathbf{P}\left(v-v_{*}\right)\left(\nabla h_{n+2}\right)\right] \cdot\left[\mathbf{P}\left(v-v_{*}\right)\left(\nabla h_{n+2}\right)_{*}\right] M M_{*} d v d v_{*} \\
+2(1-2 \varepsilon) R^{2 \gamma} \int_{\mathbb{R}^{N} \times \mathbb{R}^{N}}\left|v-v_{*}\right|^{2}\left\|\mathbf{P}\left(v-v_{*}\right)\left(\nabla h_{n+2}\right)\right\|^{2} M M_{*} d v d v_{*} \\
\quad-C_{2}(\varepsilon) \eta R^{-\gamma}\left\|h_{n+2}\langle v\rangle\right\|_{L^{2}(M)}^{2}-C_{1} R^{2(n+1)} e^{-\left(R^{n+2}-R^{n+1}\right)}\left\|h_{n+2}\right\|^{2} \\
\left.\quad-\epsilon_{2}\left(R^{n+1}-1\right)\left\|h_{n+2}\right\|_{H^{1}(M)}^{2}-C_{3} R^{2 n} e^{-\left(R^{n+1}-R^{n}\right)}\left\|h_{n+2}\right\|_{H^{1}(M)}^{2}\right]
\end{array}\right.}
\end{aligned}
$$


which writes

$$
\begin{aligned}
& \sum_{n \geq n_{0}} R^{(n+1) \gamma} D_{n}^{\mathcal{L}}(h) \geq \sum_{n \geq n_{0}} R^{(n+1) \gamma}\left[\int_{\mathbb{R}^{N} \times \mathbb{R}^{N}}\left|v-v_{*}\right|^{2} \Delta\left(h_{n+2}\right)^{2} M M_{*} d v d v_{*}\right. \\
& \quad-2\left(1-(1-2 \varepsilon) R^{2 \gamma}\right)\left\|h_{n+2}\right\|_{H^{1}(M)}^{2}-C_{1} R^{2(n+1)} e^{-\left(R^{n+2}-R^{n+1}\right)}\left\|h_{n+2}\right\|_{H^{1}(M)}^{2} \\
& \left.-C_{2}(\varepsilon) \eta R^{-\gamma}\left\|h_{n+2}\langle v\rangle\right\|_{L^{2}(M)}^{2}-\epsilon_{2}\left(R^{n+1}\right)\left\|h_{n+2}\right\|_{H^{1}(M)}^{2}-C_{3} R^{2 n} e^{-\left(R^{n+1}-R^{n}\right)}\left\|h_{n+2}\right\|_{H^{1}(M)}^{2}\right] .
\end{aligned}
$$

Now we use the explicit coercivity estimate (3.1) for Maxwell molecules to deduce that

$$
\begin{aligned}
& \sum_{n \geq n_{0}} R^{(n+1) \gamma} D_{n}^{\mathcal{L}}(h) \geq \\
& \sum_{n \geq n_{0}} R^{(n+1) \gamma}\left[\frac{3}{2} \lambda\left\|h_{n+2}\right\|_{H^{1}(M)}^{2}+\frac{3}{2} \lambda\left\|h_{n+2}\langle v\rangle\right\|_{L^{2}(M)}^{2}-6 \lambda\left\|\Pi\left(h_{n+2}\right)\right\|_{H^{1}(M)}^{2}\right. \\
& \quad-6 \lambda\left\|\Pi\left(h_{n+2}\right)\langle v\rangle\right\|_{L^{2}(M)}^{2}-2\left(1-(1-2 \varepsilon) R^{2 \gamma}\right)\left\|h_{n+2}\right\|_{H^{1}(M)}^{2} \\
& -C_{2}(\varepsilon) \eta R^{-\gamma}\left\|h_{n+2}\langle v\rangle\right\|_{L^{2}(M)}^{2}-C_{1} R^{2(n+1)} e^{-\left(R^{n+2}-R^{n+1}\right)}\left\|h_{n+2}\right\|_{H^{1}(M)}^{2} \\
& \left.\quad-\epsilon_{2}\left(R^{n+1}\right)\left\|h_{n+2}\right\|_{H^{1}(M)}^{2}-C_{3} R^{2 n} e^{-\left(R^{n+1}-R^{n}\right)}\left\|h_{n+2}\right\|_{H^{1}(M)}^{2}\right] .
\end{aligned}
$$

Since $\Pi(h)=0$, we have

$$
\begin{gathered}
6 \lambda\left\|\Pi\left(h_{n+2}\right)\right\|_{H^{1}(M)}^{2}+6 \lambda\left\|\Pi\left(h_{n+2}\right)\langle v\rangle\right\|_{L^{2}(M)}^{2} \\
=\left\|\Pi\left(h \mathbf{1}_{\left\{|\cdot| \geq R^{n+2}\right\}}\right)\right\|_{H^{1}(M)}^{2}+\left\|\Pi\left(h \mathbf{1}_{\left\{|\cdot| \geq R^{n+2}\right\}}\right)\langle v\rangle\right\|_{L^{2}(M)}^{2} \\
\leq C_{4} e^{-R^{n+2}}\|h\|_{H^{1}(\langle v\rangle \gamma M)}^{2} .
\end{gathered}
$$

Now if we choose $\varepsilon$ and $R-1>0$ small enough such that

$$
2\left(1-(1-2 \varepsilon) R^{2 \gamma}\right) \leq \frac{\lambda}{4},
$$

then $\eta$ small enough such that

$$
C_{2}(\varepsilon) \eta \leq \frac{\lambda}{4}
$$

then $n_{0}$ big enough so that $R^{n+2}(R-1) \geq \eta^{-1}$ for any $n \geq n_{0}$ (see the discussion above), and so that $R^{n+2}-R^{n+1}=R^{n+1}(R-1)$ and $R^{n+1}-R^{n}=R^{n}(R-1)$ big 
enough such that

$$
\forall n \geq n_{0}, \quad C_{1} R^{2(n+1)} e^{-\left(R^{n+2}-R^{n+1}\right)}, C_{3} R^{2 n} e^{-\left(R^{n+1}-R^{n}\right)} \leq \frac{\lambda}{4},
$$

and also $n_{0}$ big enough such that $R^{n+1}$ big enough such that

$$
\forall n \geq n_{0}, \quad \epsilon_{2}\left(R^{n+1}\right) \leq \frac{\lambda}{4}
$$

we obtain for this choice of $R, \eta$ and $n_{0}$ :

$$
\begin{aligned}
& \sum_{n \geq n_{0}} R^{(n+1) \gamma} D_{n}^{\mathcal{L}}(h) \\
& \geq \frac{\lambda}{4} \sum_{n \geq n_{0}} R^{(n+1) \gamma}\left(\left\|h_{n+2}\right\|_{H^{1}(M)}^{2}+\left\|h_{n+2}\right\|_{L^{2}\left(\langle v\rangle^{2} M\right)}^{2}\right) \\
& \quad-C_{4} \lambda\left(\sum_{n \geq n_{0}} e^{-R^{n+2}}\right)\|h\|_{H^{1}(\langle v\rangle \gamma M)}^{2} \\
& \geq\left[C_{5} R^{n_{0} \gamma}-C_{6} e^{-R^{n_{0}}}\right]\left(\|h\|_{H^{1}(\langle v\rangle \gamma M)}^{2}+\left\|h\langle v\rangle^{1+\gamma / 2}\right\|_{L^{2}(M)}^{2}\right)
\end{aligned}
$$

for some explicit constants $C_{5}, C_{6}>0$ independent on $n_{0}$. Thus by taking $n_{0}$ large enough we deduce that

$$
\sum_{n \geq n_{0}} R^{(n+1) \gamma} D_{n}^{\mathcal{L}}(h) \geq C_{7}\left(\|h\|_{H^{1}(\langle v\rangle \gamma M)}^{2}+\left\|h\langle v\rangle^{1+\gamma / 2}\right\|_{L^{2}(M)}^{2}\right)
$$

for some explicit constant $C_{7}>0$. Coming back to $D^{\mathcal{L}}(h)$, this concludes the proof of Theorem 1.3 when $\gamma<0$.

Acknowledgment: We thank François Golse for pointing us reference [8], and we thank Yan Guo and Robert Strain for useful discussions on the Landau operator, and pointing us the results in [20]. We also thank the three anonymous referees for their numerous comments and suggestions. Support by the European network HYKE, funded by the EC as contract HPRN-CT-2002-00282, is acknowledged.

\section{REFERENCES}

[1] Adams, R. A. Sobolev spaces. Pure and Applied Mathematics, Vol. 65. Academic Press, New York-London, 1975.

[2] Alexandre, R. Sur l'opérateur de Boltzmann linéaire en dimension 3 sans troncature angulaire. C. R. Acad. Sci., Paris, Sr. I, Math. 325, 9 (1997), 959-962.

[3] Alexandre, R. Remarks on 3D Boltzmann linear equation without cutoff. Transp. Theory Stat. Phys. 28, 5 (1999), 433-473. 
[4] Alexandre, R., Desvillettes, L., Villani, C., Wennberg, B. Entropy dissipation and long-range interactions. Arch. Rat. Mech. Anal. 152 (2000), 327-355.

[5] Alexandre, R., Villani, C., On the Landau approximation in plasma physics. Ann. Inst. H. Poincaré Anal. Non Linéaire 21, 1 (2004), 61-95.

[6] Arsen'ev, A. A., Buryak, O. E. On a connection between the solution of the Boltzmann equation and the solution of the Landau-Fokker-Planck equation. Math. USSR Sbornik 69, 2 (1991), 465-478.

[7] Baranger, C., Mounot, C. Explicit spectral gap estimates for the linearized Boltzmann and Landau operators with hard potentials. Rev. Mat. Iberoam. 21, 3 (2005), 819-841.

[8] Bardos, C., Caflisch, R. E., Nicolaenko, B. The Milne and Kramers problems for the Boltzmann equation of a hard sphere gas. Comm. Pure Appl. Math. 39, 3 (1986), 323-352.

[9] Bobylëv, A. V. The theory of the nonlinear spatially uniform Boltzmann equation for Maxwell molecules. Soviet Sci. Rev. Sect. C Math. Phys. Rev. Vol. 7 (1988), 111-233.

[10] Caflisch, Russel E., The Boltzmann equation with a soft potential. I. Linear, spatiallyhomogeneous. Comm. Math. Phys. 7/4 (1980), 71-95.

[11] Carleman, T. Problèmes mathématiques dans la théorie cinétique des gaz. Almqvist and Wiksells Boktryckeri Ab, Uppsala 1957.

[12] Cercignani, C. The Boltzmann equation and its applications. Springer-Verlag, New York, 1988.

[13] Cercignani, C., Illner, R., Pulvirenti, M. The mathematical theory of dilute gases. Springer-Verlag, New York, 1994.

[14] Degond, P., Lemou, M., Dispersion relations for the linearized Fokker-Planck equation. Arch. Rational Mech. Anal. 138 (1997), 137-167.

[15] Degond, P., Lucquin-Desreux, B., The Fokker-Planck asymptotics of the Boltzmann collision operator in the Coulomb case. Math. Models Methods Appl. Sci. 2, 2 (1992), 167182.

[16] Desvillettes, L., On asymptotics of the Boltzmann equation when the collisions become grazing. Transport Theory Statist. Phys. 21, 3 (1992), 259-276.

[17] Golse, F., Poupaud, F. Stationary solutions of the linearized Boltzmann equation in a half-space. Math. Methods Appl. Sci. 11 (1989), 483-502.

[18] Goudon, T. On Boltzmann equations and Fokker-Planck asymptotics: influence of grazing collision. J. Stat. Phys. 89, 3-4 (1997), 751-776.

[19] Grad, H. Asymptotic theory of the Boltzmann equation. II. Rarefied Gas Dynamics (Proc. 3rd Internat. Sympos., Palais de l'UNESCO, Paris, 1962), Vol. I, 26-59, Academic Press, New York, 1963.

[20] Guo, Y. The Landau equation in a periodic box. Comm. Math. Phys. 231 (2002), 391-434.

[21] Guo, Y. Classical solutions to the Boltzmann equation for molecules with an angular cutoff. Arch. Ration. Mech. Anal. 169 (2003), 305-353.

[22] Kato, T. Perturbation theory for linear operators. Classics in Mathematics. Springer-Verlag, Berlin, 1995.

[23] Klaus, M. Boltzmann collision operator without cut-off. Helv. Phys. Acta 50 (1977), 893-903.

[24] Landau, L. D., Lifschitz, E. M. Physical Kinetics (Course of Theoretical Physics Volume 10). Butterworth-Heinemann, 1981.

[25] Lemou, M., Linearized quantum and relativistic Fokker-Planck-Landau equations. Math. Methods Appl. Sci. 23, 12 (2000), 1093-1119. 
[26] Lions, P.-L. Regularity and compactness for Boltzmann collision operators without angular cut-off. C. R. Acad. Sci. Paris Sér. I Math. 326 (1998), 37-41.

[27] Mounot, C., Strain, R. Explicit spectral gap and coercivity estimates for the linearized Boltzmann and Landau collision operators without cutoff. Work in progress.

[28] PAO, Y.P. Boltzmann collision operator with inverse-power intermolecular potentials. I, II. Comm. Pure Appl. Math. 27 (1974), 407-428; ibid. 27 (1974), 559-581.

[29] Villani, C. On a new class of weak solutions to the spatially homogeneous Boltzmann and Landau equations. Arch. Rational Mech. Anal. 143, 3 (1998), 273-307.

[30] Villani, C. Regularity estimates via the entropy dissipation for the spatially homogeneous Boltzmann equation without cut-off. Rev. Mat. Iberoam. 15 (1999), 335-352.

[31] Villani, C. Contribution à l'étude mathématique des collisions en théorie cinétique. Univ. Paris-Dauphine France (2000).

[32] Villani, C. A Review of Mathematical Topics in Collisional Kinetic Theory. Handbook of mathematical fluid mechanics, edited by Susan Friedlander and Denis Serre, North-Holland (2002).

[33] Villani, C. Topics in Optimal Transportation. AMS, Graduate Studies in Mathematics series, vol. 58 (2003).

[34] Wang Chang, C. S. and Uhlenbeck, G. E. And de Boer, J., Studies in Statistical Mechanics, Vol. V. North-Holland, Amsterdam (1970).

C. Mounot

CEREMade, Université Paris IX Dauphine

Place du Marchal de Lattre de Tassigny

75775 Paris CEdeX 16

FRANCE

E-MAIL: cmouhot@ceremade.dauphine.fr 